\title{
Paulownia $C$-geranylated flavonoids: their structural variety, biological activity and application prospects
}

\author{
Chun-lei Cheng $\cdot$ Xian-hui Jia $\cdot$ Cheng-mei Xiao $\cdot$ Wen-zhao Tang $\mathbb{B}$
}

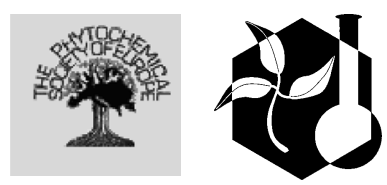

Received: 1 January 2019/Accepted: 5 June 2019/Published online: 13 June 2019

(C) Springer Nature B.V. 2019

\begin{abstract}
Paulownia species, especially their flowers and fruits, are traditionally used in Chinese herbal medicines for the treatment of infectious diseases. $C$ geranylated flavonoids were found to be the major special metabolites in Paulownia flowers and fruits, and $76 \mathrm{C}$-geranylated flavonoids had been isolated and characterized thus far. Structural variations in Paulownia C-geranylated flavonoids are mainly due to the complicated structural modifications in their geranyl substituents. These natural compounds have attracted much attention because of their various biological activities, including antioxidation, antiinflammation, cytotoxic activity and various enzymatic inhibitions, etc. Among them, diplacone, a major Paulownia component, was considered to have
\end{abstract}

\section{Cheng}

Shandong Institute for Food and Drug Control, Jinan 250101, Shandong, People's Republic of China

X. Jia $\cdot$ C. Xiao $\cdot$ W. Tang $(\square)$

Institute of Materia Medica, Shandong Academy of

Medical Sciences, No. 18877, Jingshi Road, Jinan

250062, Shandong, People's Republic of China

e-mail: twzsd@sina.com

X. Jia $\cdot$ C. Xiao $\cdot$ W. Tang

Key Laboratory for Biotech-Drugs Ministry of Health, Jinan 250062, Shandong, People's Republic of China

X. Jia $\cdot$ C. Xiao $\cdot$ W. Tang

Key Laboratory for Rare and Uncommon Diseases of Shandong Province, Jinan 250062, Shandong, People's Republic of China promise for applications in medicine. This paper summarizes the information from current publications on Paulownia $C$-geranylated flavonoids, with a focus on their structural variety, key spectroscopic characteristics, biological activity with structureactivity relationships and application prospects. We hope that this paper will stimulate further investigations of Paulownia species and this kind of natural product.

Keywords Paulownia species · $C$-geranylated flavonoids · Phytochemistry · Biological properties

\section{Introduction}

$C$-geranylated flavonoids, a small group of flavonoid derivatives consisting of a flavonoid skeleton joined with a terpenoid side-chain $\left(\mathrm{C}_{10}\right.$, geranyl substituent) via a direct carbon-carbon bond directly, are considered as the members of the prenylated flavonoids (Barron and Ibrahim 1996). Among these compounds, the geranyl has been revealed to have the potential to occur as a substituent possibly at the C-3, C-6, C-8, C-2' or C-3' positions of the flavonoid skeleton (Fig. 1). C-geranylated flavonoids, as secondary metabolic products, are biosynthesized via the mevalonate pathway (for the geranyl skeleton) and the shikimic acid pathway with cinnamoyl CoA (for the flavonoid skeleton), and the connection is 
provided by the prenyltransferases, the key biosynthetic enzymes for prenylated polyphenols in plants (Šmejkal 2014; Andersen and Markham 2006; Kuzuyama et al. 2005). Recently, an aromatic prenyltransferase was discovered in Aspergillus terreus and exhibited unprecedented promiscuity towards flavonoid acceptors to produce $C$-geranylated flavonoids (Chen et al. 2017a). $C$-geranylated flavonoids occur in relatively few plant families, such as Leguminosae, Moraceae, Rutaceae, and Ophioglossaceae (Yazaki et al. 2009). Among them, the Paulownia genus (Paulowniaceae family) is a rich natural source of this kind of compound.

The Paulownia genus was previously categorized as part of the Scrophulariaceae family, but now, it has now been categorized as a monotypic family of its own, Paulowniaceae, based on the latest molecular phylogenetic data (Erbar and Gülden 2011). Now,<smiles>[Z]C(C)=CC(C)=CCC=C(C)C</smiles>

Geranyl

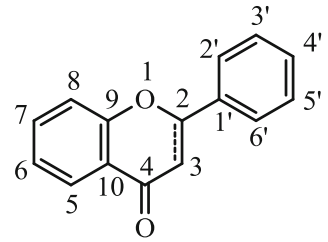

Skeleton of flavone or flavanone
Fig. 1 Structural skeleton of $C$-geranylated flavonoids obtained from Paulownia species only nine species are accepted as part of the Paulownia genus by Flora of China as shown in Table 1 (Chinese Flora Editorial Committee 1998). Paulownia species are fast-growing shade trees native to China and Southeast Asia and are mainly grown as ornamental trees or commercially for the production of hardwood timber (Zhu et al. 1986; Bergmann 1998). Moreover, their leaves, flowers, fruits and root barks are also traditionally used as Chinese herbal folk medicines for the treatment of enteritis, tonsillitis, bronchitis, dysentery, etc. (He et al. 2016). Phytochemical research has indicated that Paulownia plants contain quinones, lignans, triterpenes, phenylpropanoid glycosides, and flavonoids (Xing et al. 2013; He et al. 2016). However, $C$ geranylated flavonoids, as the main constituents, have attracted much attention due to their structural variation and assorted biological properties.

Among Paulownia species, P. tomentosa has been the most extensively investigated, and $39 \mathrm{C}$-geranylated flavanones have been isolated from its flowers and fruits (Schneiderová and Šmejkal 2014). Subsequently, another $37 C$-geranylated flavonoids (including $C$-geranylated flavanones and $C$-geranylated flavones) were further obtained from $P$. tomentosa and other Paulownia species. However, no systematic review of various aspects related to Paulownia C-geranylated flavonoids has yet been

Table 1 Paulownia plants in China

\begin{tabular}{lll}
\hline No. & Chinese name & Plant Latin name \\
\hline 1 & 楸叶泡桐 & Paulownia catalpifolia T. Gong ex D. Y. Hong \\
2 & 兰考泡桐 & Paulownia elongata S. Y. Hu \\
3 & 川泡桐 & Paulownia fargesii Franchet \\
4 & 白花泡桐 & Paulownia fortunei (Seem.) Hemsl. \\
5 & 台湾泡桐 & Paulownia kawakamii T. Itô \\
6 & 毛泡桐 & Paulownia tomentosa Steud. \\
7 & 南方泡桐 & Paulownia australis T. Gong \\
8 & 毛泡桐 (原变种) & Paulownia tomentosa var. Tomentosa \\
9 & 光泡桐 & Paulownia tomentosa var. tsinlingensis (Pai [Bai]) T. Gong \\
10 & & Paulownia coreana Uyeki ${ }^{\mathrm{b}}$ \\
\hline
\end{tabular}

The plant Latin names are presented according to the new classification (http://www.theplantlist.org), even though older classifications are presented in the references

a Status as "Synonym"

b The Paulownia species, Paulownia coreana Uyeki, investigated by Jin et al. is an unresolved name and is not accepted in Flora of China (1998) by the Chinese Flora Editorial Committee 
reported. The present paper summarizes existing publications associated with Paulownia $C$-geranylated flavonoids and their chemical structures, key spectroscopic characteristics, biological activities and application prospects. We hope that the provided information in this paper can provide an overview of research on Paulownia $C$-geranylated flavonoids and stimulate further investigations on this kind of compound.

\section{Structural variations in Paulownia $C$ - geranylflavonoids}

Seventy-six $C$-geranylflavonoids (1-76, Figs. 2, 3, 4, $5,6)$ were isolated and identified from Paulownia species and are the main bioactive constituents. Among them, $C$-geranylated flavanones represented the overwhelming majority (1-72, Figs. 2, 3, 4, 5) and only four $C$-geranylated flavones (73-76, Fig. 6) were obtained so far. Except for compounds 19 and 27, which were C-8 substituted, all the other Paulownia C-geranylated flavonoids possessed a C-6 geranyl-mono-substituted structure pattern. Paucatalinone A (19) was also the first sample as a natural dimeric $C$-geranylated flavanone derivative (Gao et al. 2015). In Paulownia C-geranylated flavanones, $\mathrm{C}-2$ in ring $\mathrm{C}$ is a chiral carbon, and most of them possess an $S$ configuration at the stereogenic centre C-2, except for compounds 19, 25, $27,37,41,43,46,60$, and 67 , which were obtained as racemic mixtures of $2 R$ and $2 S$ enantiomers. Moreover, all the stereogenic centres of $\mathrm{C}-2$ and $\mathrm{C}-3$ in Paulownia $C$-geranylated flavanonols 13, 14, 15, 22, $\mathbf{2 4}, \mathbf{3 2}, \mathbf{3 6}, \mathbf{5 1}, \mathbf{5 2}$, and 58 were $2 R, 3 R$ configurations, although 17 was a racemic mixture of $2 R, 3 R$ and $2 S, 3 S$ enantiomers.

Basically, Paulownia C-geranylated flavonoids originally occurred with an unmodified geranyl substituent, and their structural variation occurred due only to phenolic hydroxyl groups or methoxy groups in the flavonoid skeleton, such as those in compounds 1-19 (Fig. 2) and 73 (Fig. 6).

However, the geranyl substituent in Paulownia $C$ geranylflavonoids could undergo further oxidation (mainly hydroxylation or carbonylation) at different positions to give rise to various modified geranyl substituents as the linear $\mathrm{C}_{10}$ side chain (Fig. 4). It could be hydroxylated at C-7" to produce a common 7-hydroxy-3,7-dimethyl-2(E)-octenyl substituent (20-27) or a rare $(2 E, 5 E)$-7-hydroxy-3,7-dimethylocta-2,5-dien-1-yl substituent (40); at C-6" to achieve a 6-hydroxy-3,7-dimethyl-2(E)-7-octadienylsubstituent (28-37), which could be further oxidized
Fig. 2 Unmodified $C$ geranylflavanones isolated from the Paulownia genus

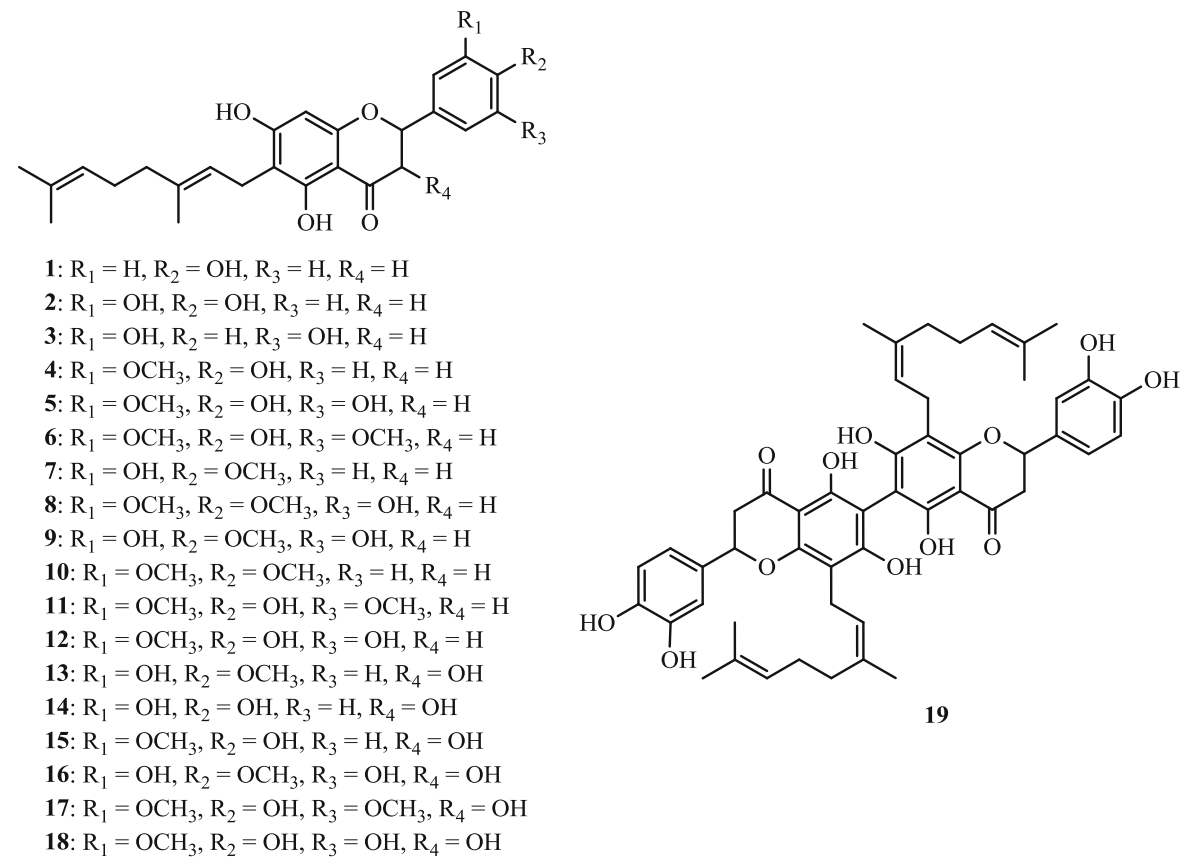


Fig. 3 Noncyclic modified $C$-geranylflavanones isolated from the Paulownia genus<smiles>[R]c1cc(C2Oc3cc(O)c(CC=C(C)CCCC(C)(C)O)c(O)c3C(=O)C2[R4])cc([R])c1[R]</smiles>

20: $\mathrm{R}_{1}=\mathrm{H}, \mathrm{R}_{2}=\mathrm{OH}, \mathrm{R}_{3}=\mathrm{H}, \mathrm{R}_{4}=\mathrm{H}$

21: $\mathrm{R}_{1}=\mathrm{OH}, \mathrm{R}_{2}=\mathrm{OH}, \mathrm{R}_{3}=\mathrm{H}, \mathrm{R}_{4}=\mathrm{H}$

22: $\mathrm{R}_{1}=\mathrm{OCH}_{3}, \mathrm{R}_{2}=\mathrm{OH}, \mathrm{R}_{3}=\mathrm{H}, \mathrm{R}_{4}=\mathrm{OH}$

23: $\mathrm{R}_{1}=\mathrm{OH}, \mathrm{R}_{2}=\mathrm{OCH}_{3}, \mathrm{R}_{3}=\mathrm{H}, \mathrm{R}_{4}=\mathrm{H}$

24: $\mathrm{R}_{1}=\mathrm{OCH}_{3}, \mathrm{R}_{2}=\mathrm{OH}, \mathrm{R}_{3}=\mathrm{H}, \mathrm{R}_{4}=\mathrm{OH}$

25: $\mathrm{R}_{1}=\mathrm{OCH}_{3}, \mathrm{R}_{2}=\mathrm{OH}, \mathrm{R}_{3}=\mathrm{OH}, \mathrm{R}_{4}=\mathrm{H}$

26: $\mathrm{R}_{1}=\mathrm{OCH}_{3}, \mathrm{R}_{2}=\mathrm{OH}, \mathrm{R}_{3}=\mathrm{OCH}_{3}, \mathrm{R}_{4}=\mathrm{H}$<smiles>[R]c1cc(C2CC(=O)c3c(O)cc(O)c(CC=C(C)CCCC(C)(C)O)c3O2)cc(C2Oc3cc(O)c(CC=C(C)CCC(O)C(=C)C)c(O)c3C(=O)C([R4])C2c2cc([R4])c([R2])c([R])c2)c1</smiles><smiles>[R]c1ccc(C2CC(=O)c3c(cc(O)c(CC(O)C(=C)CCC=C(C)C)c3O)O2)cc1[R2]</smiles>

38: $\mathrm{R}_{1}=\mathrm{H}, \mathrm{R}_{2}=\mathrm{OH}$

39: $\mathrm{R}_{1}=\mathrm{OCH}_{3}, \mathrm{R}_{2}=\mathrm{OH}$<smiles>COc1cc(C2CC(=O)c3c(cc(O)c(C/C=C(\C)C/C=C/C(C)(C)O)c3O)O2)ccc1O</smiles>

40<smiles>C=C(C)C(=O)CC/C(C)=C/Cc1c(O)cc2c(c1O)C(=O)CC(c1ccc(O)c(OC)c1)O2</smiles>

41<smiles>[R]c1cc(C2CC(=O)c3c(cc(O)c(CC=C(C)CCC(O)C([R20])(C)C)c3O)O2)cc([R])c1[R]</smiles>

42: $\mathrm{R}_{1}=\mathrm{H}, \mathrm{R}_{2}=\mathrm{OH}, \mathrm{R}_{3}=\mathrm{H}, \mathrm{R}_{4}=\mathrm{H}$

43: $\mathrm{R}_{1}=\mathrm{OCH}_{3}, \mathrm{R}_{2}=\mathrm{OH}, \mathrm{R}_{3}=\mathrm{OCH}_{3}, \mathrm{R}_{4}=\mathrm{H}$

44: $\mathrm{R}_{1}=\mathrm{H}, \mathrm{R}_{2}=\mathrm{OH}, \mathrm{R}_{3}=\mathrm{H}, \mathrm{R}_{4}=\mathrm{CH}_{3}$

45: $\mathrm{R}_{1}=\mathrm{OCH}_{3}, \mathrm{R}_{2}=\mathrm{OH}, \mathrm{R}_{3}=\mathrm{H}, \mathrm{R}_{4}=\mathrm{CH}_{3}$

46: $\mathrm{R}_{1}=\mathrm{OCH}_{3}, \mathrm{R}_{2}=\mathrm{OH}, \mathrm{R}_{3}=\mathrm{OCH}_{3}, \mathrm{R}_{4}=\mathrm{CH}_{3}$ to yield an unusual 3,7-dimethyl-6-oxoocta-2,7-dien1-yl substituent (41); or at C-2" to obtain a rare 2-hydroxy-7-methyl-3-methyleneoct-6-en-1-yl substituent (38 and 39). The oxidation could also occur at C-7" and C-8" simultaneously to form 6,7dihydroxy-3,7-dimethyloct-2-en-1-yl (42 and 43) or 6-hydroxy-7-methoxy-3,7-dimethyloct-2-en-1-yl

(44-46) substituents.

Interestingly, on the foundation of hydroxylation, further cyclic modifications could occur in the geranyl substituent to yield more attractive Paulownia $C$-geranylflavonoids (Fig. 4). The pyran or furan moiety could be generated in Paulownia $C$-geranylflavonoids by a further cyclization between the geranyl substituent and C-7 in ring A via an oxygen atom or between two carbon atoms in the geranyl via an oxygen atom. Compounds 47-56, containing a 2,3,7,8-tetrahydropyrano moiety, and compounds $\mathbf{5 7 -}$ 62, containing a 2,3-dihydropyrano moiety with a double bond assigned to C-1" and C-2", were provided by cyclization between $\mathrm{C}-3^{\prime \prime}$ of the geranyl substituent and $\mathrm{C}-7$ in ring $\mathrm{A}$ of the flavanone skeleton via an oxygen atom. Meanwhile, some other carbon atoms in these modified geranyl substituents, such as C-2" (56), C-6" (61) or C-7" (62), could be further hydroxylated to yield more complex geranylated flavonoid derivatives. Similarly, cyclization occurring between C-2" of the geranyl substituent and $\mathrm{C}-7$ in ring $\mathrm{A}$ of the flavanone skeleton via an oxygen atom could produce a $2 H$-furo unit (63 and 64), but this structural modification was not universal in natural C-geranylated flavonoid derivatives. Cyclization could also occur between two carbon atoms in the geranyl chain via an oxygen atom to 
Fig. 4 Cyclic modified $C$ geranylflavanones isolated from the Paulownia genus<smiles>[R]c1cc(C2Oc3cc4c(c(O)c3C(=O)C2[R4])CCC(C)(CCCC(C)(C)O)O4)cc([R])c1[R]</smiles>

47: $\mathrm{R}_{1}=\mathrm{OH}, \mathrm{R}_{2}=\mathrm{OH}, \mathrm{R}_{3}=\mathrm{H}, \mathrm{R}_{4}=\mathrm{H}$

48: $\mathrm{R}_{1}=\mathrm{OH}, \mathrm{R}_{2}=\mathrm{OCH}_{3}, \mathrm{R}_{3}=\mathrm{H}, \mathrm{R}_{4}=\mathrm{H}$

49: $\mathrm{R}_{1}=\mathrm{OCH}_{3}, \mathrm{R}_{2}=\mathrm{OCH}_{3}, \mathrm{R}_{3}=\mathrm{OH}, \mathrm{R}_{4}=\mathrm{H}$

50: $\mathrm{R}_{1}=\mathrm{OCH}_{3}, \mathrm{R}_{2}=\mathrm{OH}, \mathrm{R}_{3}=\mathrm{OCH}_{3}, \mathrm{R}_{4}=\mathrm{H}$

51: $\mathrm{R}_{1}=\mathrm{OCH}_{3}, \mathrm{R}_{2}=\mathrm{OH}, \mathrm{R}_{3}=\mathrm{H}, \mathrm{R}_{4}=\mathrm{OH}$

52: $\mathrm{R}_{1}=\mathrm{OH}, \mathrm{R}_{2}=\mathrm{OH}, \mathrm{R}_{3}=\mathrm{H}, \mathrm{R}_{4}=\mathrm{OH}$

53: $\mathrm{R}_{1}=\mathrm{OCH}_{3}, \mathrm{R}_{2}=\mathrm{OH}, \mathrm{R}_{3}=\mathrm{OH}, \mathrm{R}_{4}=\mathrm{H}$

54: $\mathrm{R}_{1}=\mathrm{H}, \mathrm{R}_{2}=\mathrm{OH}, \mathrm{R}_{3}=\mathrm{H}, \mathrm{R}_{4}=\mathrm{H}$

55: $\mathrm{R}_{1}=\mathrm{OCH}_{3}, \mathrm{R}_{2}=\mathrm{OH}, \mathrm{R}_{3}=\mathrm{H}, \mathrm{R}_{4}=\mathrm{H}$<smiles>[R2]CC1CC(=O)c2c(cc3c(c2O)C=CC(C)(C/C=C/C(C)(C)O)O3)O1</smiles>

62<smiles>[R2]c1ccc(C2CC(=O)c3c(cc4c(c3O)CC(C(C)(O)CCC=C(C)C)O4)O2)cc1I</smiles>

63: $\mathrm{R}_{1}=\mathrm{H}, \mathrm{R}_{2}=\mathrm{OH}$

64: $\mathrm{R}_{1}=\mathrm{OCH}_{3}, \mathrm{R}_{2}=\mathrm{OH}$<smiles></smiles>

65

56<smiles>[R]c1cc([R4])cc(C2Oc3cc4c(c(O)c3C(=O)C2[R4])C=CC(C)(CCC=C(C)C)O4)c1</smiles><smiles>COc1cc(C2CC(=O)c3c(cc(O)c(CCC4(C)CCCC(C)(C)O4)c3O)O2)cc(O)c1OC</smiles>

66

58: $\mathrm{R}_{1}=\mathrm{OCH}_{3}, \mathrm{R}_{2}=\mathrm{OH}, \mathrm{R}_{3}=\mathrm{H}, \mathrm{R}_{4}=\mathrm{OH}$

59: $\mathrm{R}_{1}=\mathrm{OCH}_{3}, \mathrm{R}_{2}=\mathrm{OH}, \mathrm{R}_{3}=\mathrm{H}, \mathrm{R}_{4}=\mathrm{H}$

60: $\mathrm{R}_{1}=\mathrm{OCH}_{3}, \mathrm{R}_{2}=\mathrm{OH}, \mathrm{R}_{3}=\mathrm{OCH}_{3}, \mathrm{R}_{4}=\mathrm{H}$<smiles>C=C(C)C(O)CCC1(C)C=Cc2c(cc3c(c2O)C(=O)CC(c2ccc(O)cc2)O3)O1</smiles>

61<smiles>C=C1CCC(O)C(C)(C)C1Cc1c(O)cc2c(c1O)C(=O)CC(c1cc(O)c(O)c(O)c1)O2</smiles>

67 yield furan (between C-3" and C-6", 65) or pyran (between C-3" and C-7", 66) moieties in Paulownia $C$-geranylflavonoids. Compound $\mathbf{6 7}$ was, to date, a unique $\mathrm{C}$-geranylflavanone with a monocyclic monoterpene side-chain isolated from Paulownia plants up to mow (Wang et al. 2019), and its oxygenated cyclogeranyl substituent was similar to that (without the hydroxyl) in some ugonins isolated from Helminthostachys zeylanica (Huang et al. 2003; 2010).

In addition, five $C$-geranylflavanones with different degraded geranyl side chains (68-72, Fig. 5) were isolated from $P$. tomentosa fruits in small amounts. Their side chains all consisted of $\mathrm{C}_{7}$ units with the decomposition of a terminational propenyl group from their geranyl substituents. These compounds were proven not to be artefacts formed during the extraction and separation procedures (Navrátilová et al. 2013).

Compared with $C$-geranylflavanones, fewer $C$-geranylated flavones were isolated from Paulownia plants. To date, only four $C$-geranylated flavones (73-76, Fig. 6) were obtained from the fruits of $P$. catalpifolia (Wang et al. 2017, 2019). The variety of their geranyl substituents was minimal, with only hydroxylation at C-7" to form a 7-hydroxy-3,7dimethyl-2(E)-octenyl substituent (74-76). 
<smiles>[R]c1cc(C2CC(=O)c3c(cc(O)c(O)c3O)O2)ccc1O</smiles><smiles>[Z]C/C=C(/C)CCC(=O)OC</smiles>

68<smiles>[Z]C/C=C(/C)CCC=O</smiles>

70<smiles>C=C(CCC(=O)OC)C(O)Cc1c(O)cc2c(c1O)C(=O)CC(c1ccc(O)cc1)O2</smiles>

Fig. 5 Degraded $C$-geranylflavanones isolated from the Paulownia genus<smiles>[R2]c1cc(-c2oc3cc(O)c(CC=C(C)CCCC(C)(C)O)c(O)c3c(=O)c2[R])cc([R4])c1[R3]</smiles>

74: $\mathrm{R}_{1}=\mathrm{H} ; \mathrm{R}_{2}=\mathrm{OH} ; \mathrm{R}_{3}=\mathrm{OH} ; \mathrm{R}_{4}=\mathrm{OCH}_{3}$

75: $\mathrm{R}_{1}=\mathrm{H} ; \mathrm{R}_{2}=\mathrm{OH} ; \mathrm{R}_{3}=\mathrm{OH} ; \mathrm{R}_{4}=\mathrm{OCH}_{3}$

76: $\mathrm{R}_{1}=\mathrm{OH} ; \mathrm{R}_{2}=\mathrm{OCH}_{3} ; \mathrm{R}_{3}=\mathrm{OH} ; \mathrm{R}_{4}=\mathrm{OCH}_{3}$

Fig. 6 -geranylated flavones isolated from the Paulownia genus

\section{Occurrence of $C$-geranylated flavonoids in Paulownia}

To date, only four Paulownia species, including $P$. tomentosa, P. catalpifolia, $P$. coreana and $P$. fortunei, have been found to contain $C$-geranylflavonoids based on different phytochemical investigations. Their flowers, leaves, and especially fruits, are an excellent source of this kind of constituent (Table 2). It has also been discovered that the glandular hairs on its young reproductive organs contain flavonoids at concentrations over 1000 times greater than those on the surfaces of its young leaves (Kobayashi et al. 2008). Asai et al. (2008) isolated a number of $C$-geranylflavanones from the viscous secretion on the surface of immature $P$. tomentosa fruits and presumed that these flavonoids were biosynthesized in the microstructures glandular trichomes on the fruit surface (Asai et al. 2008; Gang et al. 2002). In addition, the correlation between the seasonal variations and changes in the content of $C$ geranylflavonoids in $P$. tomentosa fruits has also been described, and the late autumn was considered as the appropriate harvesting time to obtain high concentrations of $C$-geranylflavonoids in $P$. tomentosa fruits (Holubová and Šmejkal 2011).

\section{Spectroscopic and structural characteristics of Paulownia $\boldsymbol{C}$-geranylflavonoids}

Two structural subtypes of Paulownia C-geranylflavonoids were isolated, $C$-geranylated flavanone and $C$-geranylated flavone. Their UV features, a main maximum absorption at approximately $\lambda 290 \mathrm{~nm}$ with a shoulder peak at approximately $\lambda 230 \mathrm{~nm}$ and a weak absorption at approximately $\lambda 340 \mathrm{~nm}$ for $C$-geranylflavanone and two main maximum UV absorption at approximately $\lambda$ 220-280 $\mathrm{nm}$ and $\lambda$ 300-400 $\mathrm{nm}$ for $C$-geranylflavone (Fig. 7), were similar to those of their respective parent flavonoid skeletons (Mabry et al. 1969). However, if the geranyl side chain was modified by the formation of a pyran ring with a double bond between C-1" and C-2", as in the structures of compounds 62 and 57-61, the conjugated chromophoric system between the pyran ring, the ring $\mathrm{A}$ and the $\mathrm{C}-4$ carbonyl group in the flavonoid skeleton caused an unusual UV spectrum with two main maximum absorptions at approximately $\lambda 230$ and $275 \mathrm{~nm}$, and a shoulder peak at approximately $\lambda 290$ and $360 \mathrm{~nm}$. This kind of modified geranyl substituent could clearly influence 
Table 2 The distribution of C-geranylated flavonoids in Paulownia species

\begin{tabular}{|c|c|c|c|c|}
\hline Compounds & Name & Species & Part & References \\
\hline \multirow[t]{3}{*}{1} & \multirow[t]{3}{*}{ Mimulone } & \multirow[t]{2}{*}{ P. tomentosa } & Fruit & $\begin{array}{l}\text { Navrátilová et al. (2013) } \\
\text { Cho et al. (2013) } \\
\text { Hanáková et al. (2015) }\end{array}$ \\
\hline & & & Flower & $\begin{array}{l}\text { Chen et al. (2009) } \\
\text { Jiang et al. (2004) }\end{array}$ \\
\hline & & P. fortunei & $\begin{array}{l}\text { Flower } \\
\text { Leaf }\end{array}$ & $\begin{array}{l}\text { Duan et al. (2007) } \\
\text { Li et al. (2008) }\end{array}$ \\
\hline \multirow[t]{5}{*}{2} & \multirow[t]{5}{*}{ Diplacone } & \multirow[t]{2}{*}{ P. tomentosa } & Fruit & $\begin{array}{l}\text { Asai et al. (2008) } \\
\text { Navrátilová et al. (2013) } \\
\text { Cho et al. (2013) } \\
\text { Ryu et al. (2017) }\end{array}$ \\
\hline & & & Flower & $\begin{array}{l}\text { Chen et al. (2009) } \\
\text { Jiang et al. (2004) }\end{array}$ \\
\hline & & P. fortunei & Flower & Duan et al. (2007) \\
\hline & & P. catalpifolia & Fruit & Wang et al. (2017) \\
\hline & & P. coreana & Flower & Jin et al. (2015) \\
\hline 3 & Schizolaenone C & P. tomentosa & Fruit & Šmejkal et al. (2010) \\
\hline \multirow[t]{2}{*}{4} & \multirow[t]{2}{*}{ 3'-O-methyldiplacone } & P. tomentosa & Fruit & $\begin{array}{l}\text { Asai et al. (2008) } \\
\text { Šmejkal et al. (2007) } \\
\text { Cho et al. (2013) } \\
\text { Hanáková et al. (2015) } \\
\text { Ryu et al. (2017) }\end{array}$ \\
\hline & & P. fortunei & Flower & Duan et al. (2007) \\
\hline 5 & 3'-O-methyl-5'-hydroxydiplacone & $\begin{array}{l}\text { P. tomentosa } \\
\text { P. coreana }\end{array}$ & $\begin{array}{l}\text { Fruit } \\
\text { Flower }\end{array}$ & $\begin{array}{l}\text { Šmejkal et al. (2008a) } \\
\text { Jin et al. (2015) }\end{array}$ \\
\hline 6 & 3'-O-methyl-5'-O-methyldiplacone & P. tomentosa & Fruit & Šmejkal et al. (2008a) \\
\hline 7 & 4'-O-methyldiplacone & P. tomentosa & $\begin{array}{l}\text { Fruit secretion } \\
\text { Fruit }\end{array}$ & $\begin{array}{l}\text { Asai et al. (2008) } \\
\text { Cho et al. (2013) }\end{array}$ \\
\hline 8 & 3',4'-O-dimethyl-5'-hydroxydiplacone & P. tomentosa & Fruit & Hanáková et al. (2015) \\
\hline 9 & $\begin{array}{l}\text { 6-Geranyl-5,7,3',5'-tetrahydroxy-4'- } \\
\text { methoxyflavanone }\end{array}$ & P. tomentosa & Fruit & Ryu et al. (2017) \\
\hline 10 & $\begin{array}{l}\text { 6-Geranyl-5,7-dihydroxy-3',4'- } \\
\text { dimethoxyflavanone }\end{array}$ & P. tomentosa & $\begin{array}{l}\text { Fruit secretion } \\
\text { Fruit }\end{array}$ & $\begin{array}{l}\text { Asai et al. (2008) } \\
\text { Hanáková et al. (2015) }\end{array}$ \\
\hline \multirow[t]{2}{*}{11} & \multirow[t]{2}{*}{$\begin{array}{l}\text { 6-Geranyl-4',5,7-trihydroxy-3',5'- } \\
\text { dimethoxyflavanone }\end{array}$} & P. tomentosa & Fruit & $\begin{array}{l}\text { Asai et al. (2008) } \\
\text { Navrátilová et al. (2013) } \\
\text { Cho et al. (2013) } \\
\text { Hanáková et al. (2015) } \\
\text { Ryu et al. (2017) }\end{array}$ \\
\hline & & P. coreana & Flower & Jin et al. (2015) \\
\hline 12 & $\begin{array}{l}\text { 6-Geranyl-4',5,5',7-tetrahydroxy-3'- } \\
\text { methoxyflavanone }\end{array}$ & P. tomentosa & $\begin{array}{l}\text { Fruit secretion } \\
\text { Fruit }\end{array}$ & $\begin{array}{l}\text { Asai et al. (2008) } \\
\text { Navrátilová et al. (2013) }\end{array}$ \\
\hline \multirow[t]{2}{*}{13} & \multirow[t]{2}{*}{$\begin{array}{l}\text { 6-Geranyl-3,3',5,7-tetrahydroxy-4'- } \\
\text { methoxyflavanone }\left(4^{\prime}-O-\right. \\
\text { methyldiplacol })\end{array}$} & P. tomentosa & $\begin{array}{l}\text { Fruit secretion } \\
\text { Fruit }\end{array}$ & $\begin{array}{l}\text { Asai et al. (2008) } \\
\text { Cho et al. (2013) } \\
\text { Ryu et al. (2017) }\end{array}$ \\
\hline & & P. fortunei & Flower & Zhang and Li (2008) \\
\hline
\end{tabular}


Table 2 continued

\begin{tabular}{|c|c|c|c|c|}
\hline Compounds & Name & Species & Part & References \\
\hline \multirow[t]{2}{*}{14} & \multirow[t]{2}{*}{ Diplacol } & P. tomentosa & Fruit secretion & Asai et al. (2008) \\
\hline & & P. coreana & Flower & Jin et al. (2015) \\
\hline \multirow[t]{8}{*}{15} & \multirow[t]{8}{*}{ 3'-O-methyldiplacol } & \multirow[t]{6}{*}{ P. tomentosa } & Fruit secretion & Asai et al. (2008) \\
\hline & & & Flower & Kobayashi et al. (2008) \\
\hline & & & \multirow[t]{4}{*}{ Fruit } & Navrátilová et al. (2013) \\
\hline & & & & Cho et al. (2013) \\
\hline & & & & Hanáková et al. (2015) \\
\hline & & & & Šmejkal et al. (2008a) \\
\hline & & P. fortunei & Flower & Zhang and Li (2008) \\
\hline & & P. coreana & Flower & Jin et al. (2015) \\
\hline 16 & $\begin{array}{l}\text { 6-Geranyl-3,3',5,5',7-pentahydroxy-4'- } \\
\text { methoxyflavane }\end{array}$ & P. tomentosa & Fruit & Cho et al. (2012) \\
\hline 17 & 3'-O-methyl-5'-methoxydiplacol & P. tomentosa & Fruit & Šmejkal et al. (2007) \\
\hline 18 & 3'-O-methyl-5'-hydroxydiplacol & P. coreana & Flower & Jin et al. (2015) \\
\hline 19 & Paucatalinone A & P. catalpifolia & Fruit & Gao et al. (2015) \\
\hline \multirow[t]{2}{*}{20} & \multirow{2}{*}{$\begin{array}{l}\text { 5,7-Dihydroxy-6-(7-hydroxy-3,7- } \\
\text { dimethyloct-2-en-1-yl)-2S-(4- } \\
\text { hydroxyphenyl)-3,4-dihydro-2H-1- } \\
\text { benzopyran-4-one }\end{array}$} & P. tomentosa & Fruit & Ryu et al. (2017) \\
\hline & & P. catalpifolia & Fruit peel & Wang et al. (2019) \\
\hline \multirow[t]{4}{*}{21} & \multirow[t]{4}{*}{ Prokinawan } & \multirow[t]{3}{*}{ P. tomentosa } & Flower & Kobayashi et al. (2008) \\
\hline & & & Fruit secretion & Asai et al. (2008) \\
\hline & & & Fruit & Ryu et al. (2017) \\
\hline & & P. catalpifolia & Fruit & Tang et al. (2017) \\
\hline 22 & $\begin{array}{l}\text { 3,3',4',5,7-Pentahydroxy-6-[7-hydroxy- } \\
\text { 3,7-dimethyl-2(E)-octenyl]flavanone }\end{array}$ & P. tomentosa & Fruit secretion & Asai et al. (2008) \\
\hline 23 & Tomentin J & P. tomentosa & Fruit & Ryu et al. (2017) \\
\hline 24 & Paucatalinone $\mathrm{J}$ & P. catalpifolia & Fruit & Wang et al. (2019) \\
\hline \multirow[t]{2}{*}{25} & \multirow[t]{2}{*}{ Isopaucatalinone B } & P. catalpifolia & Fruit & Gao et al. (2015) \\
\hline & & P. tomentosa & Fruit & Ryu et al. (2017) \\
\hline \multirow[t]{2}{*}{26} & \multirow[t]{2}{*}{ Tomentin $\mathrm{K}$} & P. tomentosa & Fruit & Ryu et al. (2017) \\
\hline & & P. catalpifolia & Fruit peel & Wang et al. (2019) \\
\hline 27 & Paucatalinone B & P. catalpifolia & Fruit & Gao et al. (2015) \\
\hline \multirow[t]{2}{*}{28} & \multirow[t]{2}{*}{ Mimulone B } & P. tomentosa & Fruit & Schneiderová et al. (2013) \\
\hline & & P. coreana & Flower & Jin et al. (2015) \\
\hline 29 & Tomentomimulol & P. tomentosa & Fruit & Schneiderová et al. (2013) \\
\hline \multirow[t]{3}{*}{30} & \multirow[t]{3}{*}{ Tanariflavanone D } & \multirow[t]{3}{*}{ P. tomentosa } & Fruit secretion & Asai et al. (2008) \\
\hline & & & Fruit & Schneiderová et al. (2013) \\
\hline & & & & Ryu et al. (2017) \\
\hline 31 & Tanariflavanone & P. tomentosa & Fruit secretion & Asai et al. (2008) \\
\hline 32 & $\begin{array}{l}(2 R, 3 R)-3,3^{\prime}, 4^{\prime}, 5,7-\text { pentahydroxy-6-[6- } \\
\text { hydroxy-3,7-dimethyl-2(E),7- } \\
\text { octadienyl]flavanone }\end{array}$ & P. tomentosa & Fruit secretion & Asai et al. (2008) \\
\hline 33 & Tomentodiplacone & P. tomentosa & Fruit & Smejkal et al. (2008a) \\
\hline \multirow[t]{2}{*}{34} & \multirow{2}{*}{$\begin{array}{l}(2 R, 3 R)-4^{\prime}, 5,5^{\prime}, 7 \text {-tetrahydroxy-3'- } \\
\text { methoxy-6-[6-hydroxy-3,7-dimethyl-2 } \\
(E), 7 \text {-octadienyl]flavanone }\end{array}$} & P. tomentosa & Fruit secretion & Asai et al. (2008) \\
\hline & & P. catalpifolia & Fruit peel & Wang et al. (2019) \\
\hline 35 & Tomentin I & P. tomentosa & Fruit & Ryu et al. (2017) \\
\hline
\end{tabular}


Table 2 continued

\begin{tabular}{|c|c|c|c|c|}
\hline Compounds & Name & Species & Part & References \\
\hline 36 & Tomentodiplacol & P. tomentosa & Fruit & Šmejkal et al. (2007) \\
\hline 37 & Paucatalinone I & P. catalpifolia & Fruit peel & Wang et al. (2019) \\
\hline 38 & Mimulone $\mathrm{H}$ & P. tomentosa & Fruit & Hanáková et al. (2015) \\
\hline 39 & Tomentodiplacone $\mathrm{M}$ & P. tomentosa & Fruit & Hanáková et al. (2015) \\
\hline 40 & Tomentodiplacone B & P. tomentosa & Fruit & $\begin{array}{l}\text { Navrátilová et al. (2013) } \\
\text { Hanáková et al. (2015) }\end{array}$ \\
\hline 41 & Tomentodiplacone I & P. tomentosa & Fruit & Navrátilová et al. (2013) \\
\hline 42 & Mimulone F & P. tomentosa & Fruit & Hanáková et al. (2015) \\
\hline 43 & Paulownione A & P. tomentosa & Fruit & Hanáková et al. (2015) \\
\hline 44 & Mimulone G & P. tomentosa & Fruit & Hanáková et al. (2015) \\
\hline 45 & Tomentodiplacone $\mathrm{G}$ & P. tomentosa & Fruit & $\begin{array}{l}\text { Navrátilová et al. (2013) } \\
\text { Hanáková et al. (2015) }\end{array}$ \\
\hline 46 & Paulownione B & P. tomentosa & Fruit & Hanáková et al. (2015) \\
\hline 47 & Tomentin A & P. tomentosa & Fruit & $\begin{array}{l}\text { Cho et al. (2013) } \\
\text { Ryu et al. (2017) }\end{array}$ \\
\hline 48 & Tomentin B & $\begin{array}{l}\text { P. tomentosa } \\
\text { P. catalpifolia }\end{array}$ & Fruit & $\begin{array}{l}\text { Cho et al. (2013) } \\
\text { Ryu et al. (2017) } \\
\text { Wang et al. (2017) }\end{array}$ \\
\hline 49 & Tomentin C & $\begin{array}{l}\text { P. tomentosa } \\
\text { P. catalpifolia }\end{array}$ & Fruit & $\begin{array}{l}\text { Cho et al. (2013) } \\
\text { Wang et al. (2017) }\end{array}$ \\
\hline 50 & Tomentin D & P. tomentosa & Fruit & $\begin{array}{l}\text { Cho et al. (2013) } \\
\text { Ryu et al. (2017) }\end{array}$ \\
\hline & & P. catalpifolia & & Wang et al. (2017) \\
\hline 51 & Tomentin E & P. tomentosa & Fruit & $\begin{array}{l}\text { Cho et al. (2013) } \\
\text { Ryu et al. (2017) }\end{array}$ \\
\hline & & P. catalpifolia & & Wang et al. (2017) \\
\hline 52 & Tomentin F & P. tomentosa & Fruit & Ryu et al. (2017) \\
\hline 53 & Tomentin G & P. tomentosa & Fruit & Ryu et al. (2017) \\
\hline 54 & Tomentin $\mathrm{H}$ & $\begin{array}{l}\text { P. tomentosa } \\
\text { P. catalpifolia }\end{array}$ & $\begin{array}{l}\text { Fruit } \\
\text { Fruit peel }\end{array}$ & $\begin{array}{l}\text { Ryu et al. (2017) } \\
\text { Wang et al. (2019) }\end{array}$ \\
\hline 55 & Paucatalinone G & P. catalpifolia & Fruit peel & Wang et al. (2019) \\
\hline 56 & Tomentodiplacone $\mathrm{N}$ & P. tomentosa & Fruit & Hanáková et al. (2015) \\
\hline 57 & Tomentone & P. tomentosa & Fruit & Hanáková et al. (2015) \\
\hline 58 & Tomentodiplacol B & P. tomentosa & Fruit & Hanáková et al. (2015) \\
\hline 59 & Tomentodiplacone $\mathrm{O}$ & P. tomentosa & Fruit & Hanáková et al. (2017) \\
\hline 60 & Paulownione $\mathrm{C}$ & P. tomentosa & Fruit & Hanáková et al. (2017) \\
\hline 61 & Mimulone C & P. tomentosa & Fruit & Navrátilová et al. (2013) \\
\hline 62 & Mimulone D & P. tomentosa & Fruit & Navrátilová et al. (2013) \\
\hline 63 & Bonannione B & P. tomentosa & Fruit & Hanáková et al. (2015) \\
\hline 64 & Tomentodiplacone L & P. tomentosa & Fruit & Hanáková et al. (2015) \\
\hline 65 & Tomentodiplacone $\mathrm{H}$ & P. tomentosa & Fruit & Navrátilová et al. (2013) \\
\hline 66 & Paucatalinone $\mathrm{H}$ & P. catalpifolia & Fruit peel & Wang et al. (2019) \\
\hline 67 & Paucatalinone F & P. catalpifolia & Fruit peel & Wang et al. (2019) \\
\hline 68 & Tomentodiplacone C & P. tomentosa & Fruit & Navrátilová et al. (2013) \\
\hline 69 & Tomentodiplacone D & P. tomentosa & Fruit & Navrátilová et al. (2013) \\
\hline
\end{tabular}


Table 2 continued

\begin{tabular}{lllll}
\hline Compounds & Name & Species & Part & References \\
\hline $\mathbf{7 0}$ & Tomentodiplacone E & P. tomentosa & Fruit & Navrátilová et al. (2013) \\
$\mathbf{7 1}$ & Tomentodiplacone F & P. tomentosa & Fruit & Navrátilová et al. (2013) \\
$\mathbf{7 2}$ & Mimulone E & P. tomentosa & Fruit & Navrátilová et al. (2013) \\
$\mathbf{7 3}$ & Paucatalinone C & P. catalpifolia & Fruit & Wang et al. (2017) \\
$\mathbf{7 4}$ & Paucatalinone D & P. catalpifolia & Fruit & Wang et al. (2017) \\
$\mathbf{7 5}$ & Paucatalinone E & P. catalpifolia & Fruit & Wang et al. (2017) \\
$\mathbf{7 6}$ & Paucatalinone K & P. catalpifolia & Fruit peel & Wang et al. (2019) \\
\hline
\end{tabular}

the UV spectrum obviously (Hanáková et al. 2015, 2017).

In $C$-geranylated flavanone, the chiral carbon of $\mathrm{C}-2$ in ring $\mathrm{C}$ produces different Cotton effects in its CD spectrum at $\lambda 320-360 \mathrm{~nm}$ and $\lambda 280-310 \mathrm{~nm}$ (Slade et al. 2005). Most of the Paulownia $C$ geranylated flavanones possessed a $2 S$ absolute configuration, which could be deduced by a positive effect at approximately $330 \mathrm{~nm}$ and a negative effect at approximately $290 \mathrm{~nm}$. However, no obvious Cotton effects were found in their $C D$ spectra of some racemic mixtures of $2 R$ and $2 S$ enantiomers (Fig. 8).

In addition, the key ESI-MS/MS characteristic of Paulownia $C$-geranylated flavonoids under the positive ion model was different from that under the negative ion model. In the positive ESI-MS/MS test, quasi-molecular ion peaks such as $[\mathrm{M}+\mathrm{H}]^{+}$and/or $[\mathrm{M}+\mathrm{Na}]^{+}$appeared in the ESI-MS ${ }^{1}$ spectrum. The quasi-molecular ion peak was subjected to $\mathrm{MS}^{2}$ (ESIMS/MS) analysis, and the main positive fragment ion peak of the parent flavonoid structure with a methylene was generated by the neutral loss of a $\mathrm{C}_{9}$ unit from the $\mathrm{C}_{10}$ side chain in the $C$-geranylated flavonoid by further fragmentation (Hsu et al. 2011; Lin et al. 2012). However, as measured by the negative ESI-MS/MS, the quasi-molecular ion peak as $[\mathrm{M}-\mathrm{H}]^{-}$was generated in the ESI-MS ${ }^{1}$ spectrum, and the main negative ion peak of the parent flavonoid structure fragment was generated in the ESI-MS $^{2}$ spectrum by the neutral loss of the whole $\mathrm{C}_{10}$ side chain in further fragmentation. The positive and negative ESI-MS/MS assays of diplacone (2), a major Paulownia $C$-geranylflavanone, corroborated the above difference (Fig. 9). Furthermore, the UV and ESI-MS/MS features of $C$-geranylated flavonoids could be used to distinguish this kind of natural constituent. Tang et al. (2017) attempted to detect $C$ geranylated flavonoids in the fruits of $P$. catalpifolia by HPLC-DAD-ESI-MS/MS coupling techniques, unfortunately, accurate structures for each detected HPLC signal could not be demonstrated definitively only by UV and MS experiments alone because of the above uncertainties.

\section{Biological activities of Paulownia $C$-geranylated flavonoids}

Antioxidant effects

Paulownia C-geranylated flavonoids are natural polyphenols, and antioxidant activity is their most basic biological activity, involved in ageing, inflammation, cancer, diabetes, and neurodegenerative diseases, is very important for human health protection.

In general, Paulownia $C$-geranylated flavonoids possessed very good free radical quenching activity, such as scavenging DPPH (Table 3), due to their ortho-dihydroxy functionality in the $\mathrm{B}$ ring. The geranyl side chain did not affect activity significantly, but it could modify the solubility of Paulownia C-geranylated flavonoids, such as diplacone (2), and eventually affected their reaction kinetics (Smejkal et al. 2007; Zima et al. 2010; Asai et al. 2008; Wang et al. 2017). However, Paulownia C-geranylated flavonoids revealed different cellular cytoprotective effects on different cell lines damaged by diverse oxidants in vitro (Table 3). Interestingly, diplacone (2) and its unmodified $C$-geranylated flavanone analogues, 3'-O-methyl-5'-hydroxydiplacone (5), 3'- 


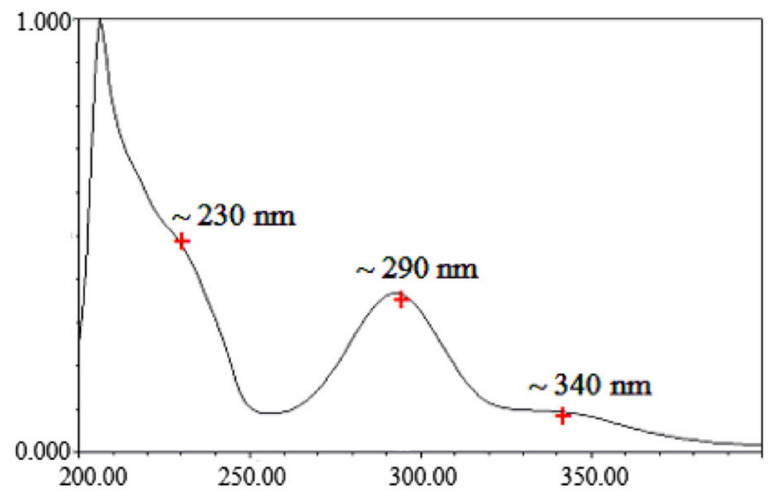

(a)

Fig. $7 \mathrm{UV}$ spectra for $C$-geranylated flavanone and $C$ geranylated flavone. The UV spectra of $C$-geranylated flavanone and $C$-geranylated flavone could be described by diplacone (2) and paucatalinone $C$ (73) as samples, respectively. a UV spectrum of diplacone (2) as a $C$-geranylated flavanone with a main maximum absorption at approximately

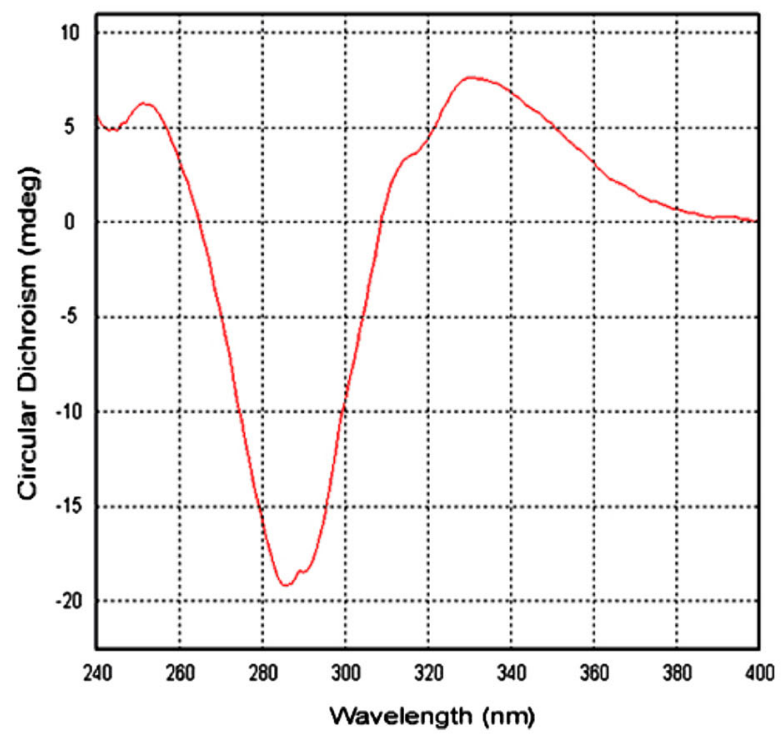

(a)

Fig. 8 Typical CD spectrum of $2 S$ configurational isomer and racemic mixtures of $2 R$ and $2 S$ enantiomers. The typical $C D$ spectrum of $2 S$ configurational isomer and racemic mixtures of $2 R$ and $2 S$ enantiomers could be described by paucatalinone $\mathrm{H}$ (66) and paucatalinone $\mathrm{F}(\mathbf{6 7})$ as samples, respectively. a CD

$O$-methyl-5'-O-methyldiplacone (6) and $3^{\prime}-O-$ methyldiplacol (15), could significantly decreas the levels of reactive oxygen species and cellular DNA damage in 2 Gy-irradiated AHH-1 cells (Moon et al. 2014). Diplacone (2), paucatalinone A (19) and paucatalinone C (73) also obviously protected

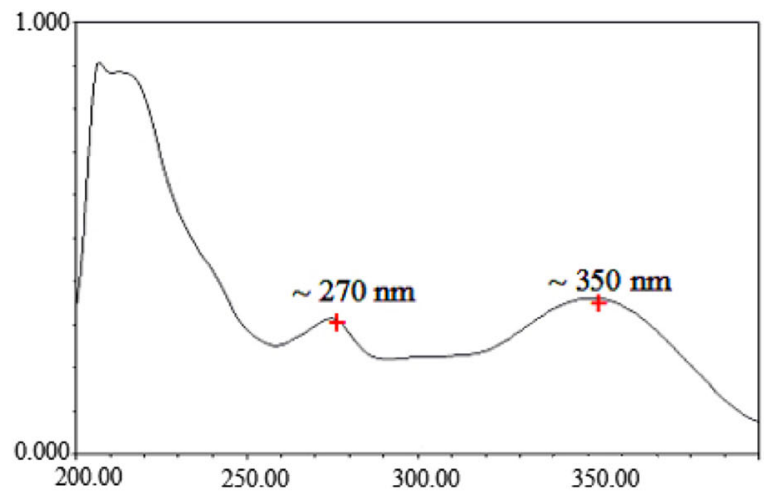

(b)

$\lambda 290 \mathrm{~nm}$, a shoulder peak at approximately $\lambda 230 \mathrm{~nm}$ and a weak absorption at approximately $\lambda 340 \mathrm{~nm}$. b UV spectrum of paucatalinone $C(\mathbf{7 3})$ as a $C$-geranylated flavone with two main maximum UV absorption at approximately $\lambda 270 \mathrm{~nm}$ and $\lambda$ $350 \mathrm{~nm}$

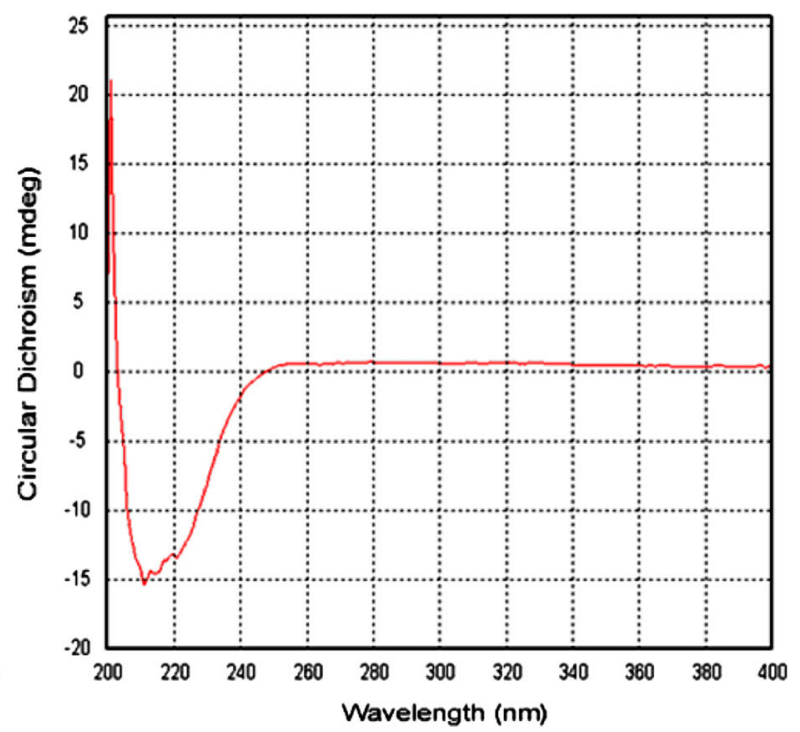

(b)

spectrum of paucatalinone $\mathrm{H}$ (66) as a $2 S$ configurational isomer with a positive Cotton effect at approximately $328 \mathrm{~nm}$ and a negative effect at approximately $285 \mathrm{~nm}$. b CD spectrum of paucatalinone $\mathrm{F}(67)$ as a racemic mixtures of $2 R$ and $2 S$ enantiomers with no obvious Cotton effects

premature senescent human embryonic lung diploid fibroblast cells at $10 \mu \mathrm{M}$ from ageing induced by $\mathrm{H}_{2} \mathrm{O}_{2}$ (Tang et al. 2017; Wang et al. 2017). Thirteen $C$-geranylated flavonoids isolated from $P$. catalpifolia had been evaluated for their antioxidant activity on HUVEC injury induced by homocysteine or $\mathrm{H}_{2} \mathrm{O}_{2}$, 

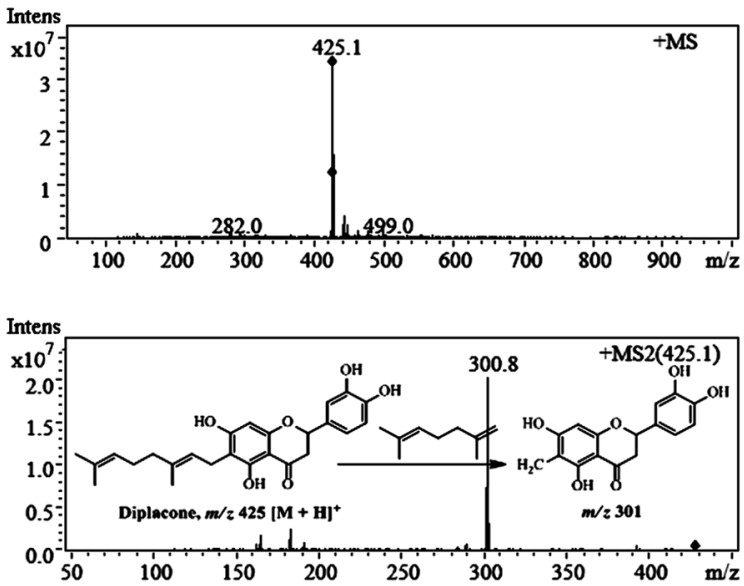

(a)

Fig. 9 Key ESI-MS/MS characteristics of Paulownia Cgeranylated flavonoids in positive ion mode and negative ion mode. Key ESI-MS/MS characteristics of Paulownia $C$ geranylated flavonoids could be described with diplacone (2) as an example. a: Main ESI-MS/MS characteristics of $\mathbf{2}$ in the positive ion mode. $\mathrm{m} / \mathrm{z} 425.1$ is the quasi-molecular ion peak as $[\mathrm{M}+\mathrm{H}]^{+}$, and $m / z 300.8$ is the fragment ion peak of the parent

and compounds 2, 25, 26, 34 and 67 caused improved proliferative activity at $10 \mu \mathrm{M}$, however, at $20 \mu \mathrm{M}$ or higher, diplacone (2) expressed cytotoxic activity to HUVECs and reduced its proliferative activity (Chen et al. 2017b; Wang et al. 2019).

\section{Anti-inflammatory effects}

For Paulownia $C$-geranylated flavonoids, only mimulone (1) and diplacone (2) were evaluated in vivo in a colitis model in Wistar rats. When orally administered at a bolus dose of $25 \mathrm{mg} / \mathrm{kg}$, they all exhibited greater effects than the positive control of sulfasalazine, reducing the level of COX-2 and increasing the ratio of pro-MMP2/MMP2 by prophylactic/therapeutic administration (Vochyánová et al. 2015).

Moreover, different pro-inflammatory and antiinflammatory cytokines produced by various inflammatory cell models were detected in vitro to evaluate the anti-inflammatory effects of Paulownia C-geranylated flavonoids (Table 4). Diplacone (2) significantly downregulated the expression of tumour necrosis factor $\alpha(\mathrm{TNF}-\alpha)$ and monocyte chemoattractant protein 1 (MCP-1) and upregulated the expression of zinc finger protein 36 (ZFP36) at
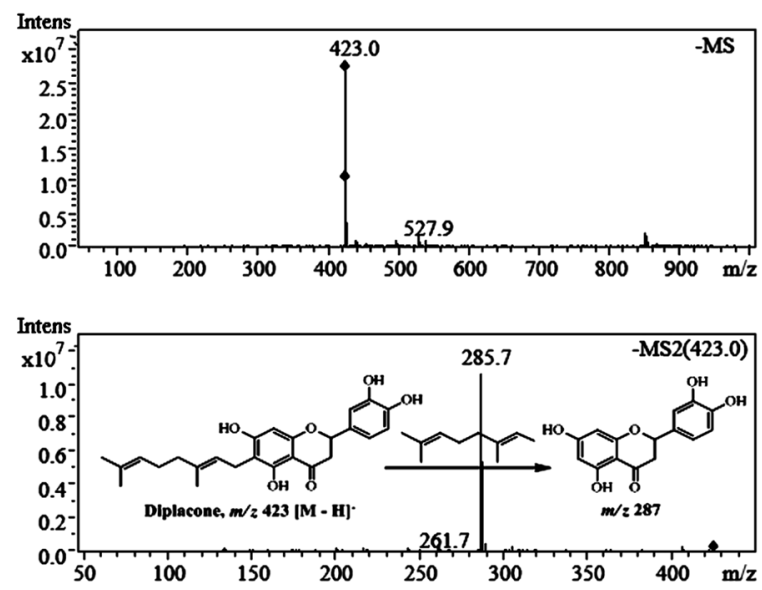

(b)

flavonoid structure with a methylene, which was produced by a neutral loss of a $\mathrm{C}_{9}$ unit from the $\mathrm{C}_{10}$ side chain in $\mathbf{2}$ by further fragmentation. b: Main ESI-MS/MS characteristics of $\mathbf{2}$ in the negative ion mode. $m / z 423.0$ is the quasi-molecular ion peak as $[\mathrm{M}-\mathrm{H}]^{-}$, and $m / z 285.7$ is the fragment ion peak of the parent flavonoid structure, which was produced by the neutral loss of the whole $\mathrm{C}_{10}$ side chain in $\mathbf{2}$ by further fragmentation

$10 \mu \mathrm{M}$, with effects even better than those of the positive control of indomethacin (Hošek et al. 2010), which also inhibited I $\mathrm{kB}-\alpha$ degradation and COX-2 expression at $1.25 \mu \mathrm{M}$ in the murine macrophage cell line J774.A1 (Hošek et al. 2013). Compounds 2, 5, 11, 14, 15, and 17 exhibited significant inhibition of LPS-induced NO production in murine macrophage RAW264.7 cells with $\mathrm{IC}_{50}$ values from 1.48 to $16.66 \mu \mathrm{M}$, compared with $\mathrm{IC}_{50} 16.60 \mu \mathrm{M}$ of aminoguanidine as the positive control (Jin et al. 2015). In addition, the effects of 21 Paulownia $C$ geranylated flavonoids on the secretion of the typical pro-inflammatory cytokine TNF- $\alpha$ in LPS-stimulated THP-1 cell from were evaluated. Compounds $2, \mathbf{4}, \mathbf{8}$, 38 and 56 could affect the secretion of TNF- $\alpha$ at $2.0 \mu \mathrm{M}$ more than prednisone as the positive control, and mimulone $\mathrm{H}$ (38) and tomentodiplacone $\mathrm{N}(\mathbf{5 6})$ significantly increased the level of reactive oxygen species (ROS) in the THP-1 cells without LPS stimulation (Hanáková et al. 2015). In another biological activity assay on human alveolar basal epithelial cells (A549 cells), compounds 23, 26, 35, 52-54 significantly inhibited TNF- $\alpha$-induced IL-8 levels at a concentration of $2.5 \mu \mathrm{M}$ without detectable cell toxicity (Ryu et al. 2017). 
Table 3 Antioxidant activity of different Paulownia C-geranylated flavonoids in comparison to standard antioxidants

\begin{tabular}{|c|c|c|c|c|}
\hline Compounds & DPPHquenchingactivity & $\begin{array}{l}\text { Cytoprotective effect } \\
\text { (cell viability } \% \text { at } 10 \mu \mathrm{M} \text { ) }\end{array}$ & Activity of the positive control & References \\
\hline \multirow[t]{2}{*}{1} & TEAC $0.4 \pm 0.004$ at $10 \mu \mathrm{M}^{\mathrm{a}}$ & & $\mathrm{NP}^{\mathrm{b}}$ & Šmejkal et al. (2007) \\
\hline & TEAC $0.02 \pm 0.01$ at $50 \mu \mathrm{M}$ & & NP & Zima et al. (2010) \\
\hline \multirow[t]{4}{*}{2} & TEAC $5.2 \pm 0.001$ at $10 \mu \mathrm{M}$ & & NP & Šmejkal et al. (2007) \\
\hline & TEAC $1.06 \pm 0.04$ at $50 \mu \mathrm{M}$ & & NP & Zima et al. (2010) \\
\hline & $\mathrm{SC}_{50} 3.2 \mu \mathrm{g} / \mathrm{mL}^{\mathrm{c}}$ & & $\alpha$-Tocopherol $\left(\mathrm{SC}_{50} 4.7 \mu \mathrm{g} / \mathrm{mL}\right)$ & Asai et al. (2008) \\
\hline & & HUVECs $(72.7 \%)^{\mathrm{e}}$ & NP & Wang et al. (2019) \\
\hline \multirow[t]{3}{*}{4} & TEAC $0.8 \pm 0.006$ at $10 \mu \mathrm{M}$ & & NP & Šmejkal et al. (2007) \\
\hline & TEAC $0.12 \pm 0.02$ at $50 \mu \mathrm{M}$ & & NP & Zima et al. (2010) \\
\hline & $\mathrm{SC}_{50} 3.2 \mu \mathrm{g} / \mathrm{mL}$ & & $\alpha$-Tocopherol $\left(\mathrm{SC}_{50} 4.7 \mu \mathrm{g} / \mathrm{mL}\right)$ & Asai et al. (2008) \\
\hline 5 & TEAC $0.98 \pm 0.03$ at $50 \mu \mathrm{M}$ & & NP & Zima et al. (2010) \\
\hline 6 & TEAC $0.29 \pm 0.02$ at $50 \mu \mathrm{M}$ & & NP & Zima et al. (2010) \\
\hline 10 & $\mathrm{SC}_{50}>50 \mu \mathrm{g} / \mathrm{mL}$ & & $\alpha$-Tocopherol $\left(\mathrm{SC}_{50} 4.7 \mu \mathrm{g} / \mathrm{mL}\right)$ & Asai et al. (2008) \\
\hline 11 & $\mathrm{SC}_{50}>50 \mu \mathrm{g} / \mathrm{mL}$ & & $\alpha$-Tocopherol $\left(\mathrm{SC}_{50} 4.7 \mu \mathrm{g} / \mathrm{mL}\right)$ & Asai et al. (2008) \\
\hline 12 & $\mathrm{SC}_{50} 4.5 \mu \mathrm{g} / \mathrm{mL}$ & & $\alpha$-Tocopherol $\left(\mathrm{SC}_{50} 4.7 \mu \mathrm{g} / \mathrm{mL}\right)$ & Asai et al. (2008) \\
\hline 14 & $\mathrm{SC}_{50} 1.9 \mu \mathrm{g} / \mathrm{mL}$ & & $\alpha$-Tocopherol $\left(\mathrm{SC}_{50} 4.7 \mu \mathrm{g} / \mathrm{mL}\right)$ & Asai et al. (2008) \\
\hline 15 & TEAC $0.10 \pm 0.00$ at $50 \mu \mathrm{M}$ & & $\mathrm{NP}$ & Zima et al. (2010) \\
\hline 20 & & HUVECs $(56.8 \%)$ & NP & Wang et al. (2019) \\
\hline 21 & $\mathrm{SC}_{50} 1.9 \mu \mathrm{g} / \mathrm{mL}$ & & $\alpha$-Tocopherol $\left(\mathrm{SC}_{50} 4.7 \mu \mathrm{g} / \mathrm{mL}\right)$ & Asai et al. (2008) \\
\hline 22 & $\mathrm{SC}_{50} 1.8 \mu \mathrm{g} / \mathrm{mL}$ & & $\alpha$-Tocopherol $\left(\mathrm{SC}_{50} 4.7 \mu \mathrm{g} / \mathrm{mL}\right)$ & Asai et al. (2008) \\
\hline 24 & & HUVECs $(56.9 \%)$ & NP & Wang et al. (2019) \\
\hline 25 & & HUVECs $(69.1 \%)$ & NP & Wang et al. (2019) \\
\hline 26 & & HUVECs $(70.0 \%)$ & NP & Wang et al. (2019) \\
\hline 32 & $\mathrm{SC}_{50} 2.9 \mu \mathrm{g} / \mathrm{mL}$ & & $\alpha$-Tocopherol $\left(\mathrm{SC}_{50} 4.7 \mu \mathrm{g} / \mathrm{mL}\right)$ & Asai et al. (2008) \\
\hline 33 & TEAC $0.14 \pm 0.04$ at $50 \mu \mathrm{M}$ & & NP & Zima et al. (2010) \\
\hline \multirow[t]{2}{*}{34} & $\mathrm{SC}_{50} 1.8 \mu \mathrm{g} / \mathrm{mL}$ & & $\alpha$-Tocopherol $\left(\mathrm{SC}_{50} 4.7 \mu \mathrm{g} / \mathrm{mL}\right)$ & Asai et al. (2008) \\
\hline & & HUVECs $(80.6 \%)$ & NP & Wang et al. (2019) \\
\hline 36 & TEAC $2.0 \pm 0.007$ at $10 \mu \mathrm{M}$ & & NP & Šmejkal et al. (2007) \\
\hline 37 & & HUVECs $(70.3 \%)$ & NP & Wang et al. (2019) \\
\hline 40 & TEAC $0.12 \pm 0.00$ at $50 \mu \mathrm{M}$ & & NP & Zima et al. (2010) \\
\hline 51 & & HUVECs $(55.4 \%)$ & NP & Wang et al. (2019) \\
\hline 54 & & HUVECs $(56.1 \%)$ & NP & Wang et al. (2019) \\
\hline 55 & & HUVECs $(69.6 \%)$ & NP & Wang et al. (2019) \\
\hline 66 & & HUVECs $(59.1 \%)$ & NP & Wang et al. (2019) \\
\hline 67 & & HUVECs $(78.4 \%)$ & NP & Wang et al. (2019) \\
\hline \multirow[t]{2}{*}{73} & $\mathrm{IC}_{50} 4.82 \mu \mathrm{M}^{\mathrm{d}}$ & & $\alpha$-Tocopherol $\left(\mathrm{IC}_{50} 12.11 \mu \mathrm{M}\right)$ & Wang et al. (2017) \\
\hline & & 2BS cells $(83.26 \%)^{\mathrm{f}}$ & $\alpha$-Tocopherol (74.55\%) & Wang et al. (2017) \\
\hline \multirow[t]{2}{*}{74} & $\mathrm{IC}_{50} 5.15 \mu \mathrm{M}$ & & $\alpha$-Tocopherol $\left(\mathrm{IC}_{50} 12.11 \mu \mathrm{M}\right)$ & Wang et al. (2017) \\
\hline & & 2BS cells $(61.04 \%)$ & $\alpha$-Tocopherol (74.55\%) & Wang et al. (2017) \\
\hline \multirow[t]{2}{*}{75} & $\mathrm{IC}_{50} 15.22 \mu \mathrm{M}$ & & $\alpha$-Tocopherol $\left(\mathrm{IC}_{50} 12.11 \mu \mathrm{M}\right)$ & Wang et al. (2017) \\
\hline & & 2BS cells $(58.13 \%)$ & $\alpha$-Tocopherol (74.55\%) & Wang et al. (2017) \\
\hline
\end{tabular}


Table 3 continued

\begin{tabular}{lccc}
\hline Compounds DPPHquenchingactivity & $\begin{array}{l}\text { Cytoprotective effect } \\
\text { (cell viability } \% \text { at } 10 \mu \mathrm{M})\end{array}$ & Activity of the positive control & References \\
\hline $\mathbf{7 6}$ & HUVECs $(58.2 \%)$ & NP & Wang et al. (2019) \\
\hline a TEAC: Trolox equivalent antioxidant capacity & \\
b NP: data are not provided & \\
${ }^{\text {c }}$ EC 50 : half maximal effective concentration & \\
${ }^{\text {d }}$ IC 5 : inhibitory concentration & \\
e HUVECs: human umbilical vein endothelial cells & \\
f $2 B S$ cells: senescent human embryonic lung diploid fibroblasts cells &
\end{tabular}

Cytotoxic activities

The cytotoxic activities of some Paulownia $C$ geranylated flavonoids were tested in different cell lines (Table 5). Compounds $\mathbf{2}-\mathbf{5}$ were all active $\left(\mathrm{EC}_{50}<10 \mu \mathrm{M}\right)$ against breast carcinoma (MCF-7), T-lymphoblastic leukaemia (CEM), multiple myeloma (RPMI 8226 and U266), cervical cancer cells (HeLa), monocytic leukaemia cell lineTHP-1, and the normal BJ fibroblast cell line (Šmejkal et al. 2010). Moreover, compounds 1, 6, 8, 10, 15, 39, 56, and 64 also showed potential cytotoxic effects all with $\mathrm{IC}_{50}$ values $<10 \mu \mathrm{M}$ on the viability of THP-1 cells (Hanáková et al. 2015). In addition, paucatalinone A (19) displayed good antiproliferative effects on human lung cancer cells $\mathrm{A} 549\left(\mathrm{IC}_{50} 8.9 \mu \mathrm{M}\right)$ with a clear increase in the percentage of cells in G1 phase and a decrease in the percentage of cells in S and G2/ $\mathrm{M}$ phases in comparison with the control cells (Gao et al. 2015). Tomentodiplacone B (40) could inhibit human monocytic leukaemia (THP-1) cell growth in a dose-dependent manner at concentrations of $5 \mu \mathrm{M}$ or higher by directly inhibiting the cyclin-dependent kinase 2 signal pathway as a possible mechanisms (Kollár et al. 2011).

\section{Antibacterial effects}

Antimicrobial assays for some Paulownia C-geranylated flavonoids were carried out, and the results indicated that Gram-positive bacteria were susceptible to this kind of natural product, however, none of the compounds was able to inhibit the growth of gram-negative bacteria or the yeast. Compounds 1, 2, 4, 5, 6, 15 and 33 exhibited positive activity in the range of the concentrations tested for Bacillus, Enterococcus, Listeria, Staphylococcus strains and 15 was the most active in MICs of $2-4 \mu \mathrm{g} \mathrm{mL}$ (Šmejkal et al. 2008a, b). Furthermore, compounds 1 and $\mathbf{1 5}$ were also active to various methicillin resistant strains of $S$. aureus (MRSA) with promising anti-MRSA activity (Ríos and Recio 2005). Compound 40 inhibited the growth of different MRSA bacteria with MICs in the range of $8-16 \mu \mathrm{g} \mathrm{mL}^{-1}$ (Navrátilová et al. 2013; 2016).

Antiparasitic activities

The antiparasitic activities of Paulownia C-geranylated flavonoids mainly involved the effects of seven unmodified $C$-geranylflavanones on Leishmania species, including mimulone (1), diplacone (2), 3'-Omethyldiplacone (4), 3'-O-methyl-5'-hydroxydiplacone (5), 3'-O-methyl-5'-O-methyldiplacone (6), 4'$O$-methyldiplacone (7), and $3^{\prime}$-O-methyldiplacol (15). Compounds 4 and 6 achieved significant antileishmanial activity with $\mathrm{IC}_{50}$ values of 10.4 and $12.7 \mu \mathrm{M}$ against $L$. donovani, and 11.3 and $8.0 \mu \mathrm{M}$ against L. braziliensis, respectively, compared with 9.5 and $6.7 \mu \mathrm{M}$ of miltefosine as the positive control (Navrátilová et al. 2016). Furthermore, diplacone (2) was also active ( $\mathrm{IC}_{50}$ $1.4 \mu \mathrm{g} \mathrm{mL}^{-1}$ ) against the related kinetoplastid parasite Trypanosoma brucei brucei (Salem et al. 2011).

Enzymatic inhibitory effects

Different kinds of enzymatic assays in vitro were explored for the biological screening of Paulownia C-geranylated flavonoids (Table 6). 
Table 4 Anti-inflammatory effects of different Paulownia $C$-geranylated flavonoids in comparison to the positive controls

\begin{tabular}{|c|c|c|c|}
\hline Compounds & Anti-inflammatory effects ${ }^{\mathrm{a}, \mathrm{b}}$ & Positive control & References \\
\hline 1 & $\begin{array}{l}\text { Colitismodel induced in Wistar rats (ameliorated the symptoms of colitis; } \\
\text { reduced the levels of COX-2; increased the ratio of pro-MMP2/MMP2) }\end{array}$ & Sulfasalazine & $\begin{array}{l}\text { Vochyánová } \\
\text { et al. (2015) }\end{array}$ \\
\hline \multirow[t]{5}{*}{2} & $\begin{array}{l}\text { Colitismodel induced in Wistar rats (ameliorated the symptoms of colitis; } \\
\text { reduced the levels of COX-2; increased the ratio of pro-MMP2/MMP2) }\end{array}$ & Sulfasalazine & $\begin{array}{l}\text { Vochyánová } \\
\text { et al. (2015) }\end{array}$ \\
\hline & ${ }^{\mathrm{c}}$ LPS-induced NO production in RAW264.7 cells $\left(\mathrm{IC}_{50} 5.02 \mu \mathrm{M}\right)$ & $\begin{array}{l}\text { Aminoguanidine } \\
\qquad\left(\mathrm{IC}_{50} 16.60 \mu \mathrm{M}\right)\end{array}$ & Jin et al. (2015) \\
\hline & $\begin{array}{l}\text { LPS-induced murine macrophage cell lineJ774.A1 (ROS level; IкB- } \alpha \\
\text { degradation; COX-2 expression) }\end{array}$ & NP & $\begin{array}{l}\text { Hošek et al. } \\
\text { (2013) }\end{array}$ \\
\hline & $\begin{array}{l}\text { LPS-induced THP-1 cells (TNF- } \alpha \text { expression; MCP-1 expression; ZFP36 } \\
\text { expression) }\end{array}$ & Indomethacin & $\begin{array}{l}\text { Hošek et al. } \\
\quad(2010)\end{array}$ \\
\hline & $\begin{array}{l}\text { LPS-induced THP-1 cells (TNF- } \alpha \text { expression; nuclear translocation of NF- } \kappa B \text {; } \\
\text { generation of ROS) }\end{array}$ & Prednisone & $\begin{array}{l}\text { Hanáková et al. } \\
\text { (2015) }\end{array}$ \\
\hline 4 & $\begin{array}{l}\text { LPS-induced THP-1 cells (TNF- } \alpha \text { expression; nuclear translocation of NF- } \kappa B \text {; } \\
\text { generation of ROS) }\end{array}$ & Prednisone & $\begin{array}{l}\text { Hanáková et al. } \\
\text { (2015) }\end{array}$ \\
\hline 5 & LPS-induced NO production in RAW264.7 cells $\left(\mathrm{IC}_{50} 1.48 \mu \mathrm{M}\right)$ & $\begin{array}{l}\text { Aminoguanidine } \\
\qquad\left(\mathrm{IC}_{50} 16.60 \mu \mathrm{M}\right)\end{array}$ & Jin et al. (2015) \\
\hline 11 & LPS-induced NO production in RAW264.7 cells $\left(\mathrm{IC}_{50} 6.44 \mu \mathrm{M}\right)$ & $\begin{array}{l}\text { Aminoguanidine } \\
\qquad\left(\mathrm{IC}_{50} 16.60 \mu \mathrm{M}\right)\end{array}$ & Jin et al. (2015) \\
\hline 14 & LPS-induced NO production in RAW264.7 cells $\left(\mathrm{IC}_{50} 4.53 \mu \mathrm{M}\right)$ & $\begin{array}{l}\text { Aminoguanidine } \\
\qquad\left(\mathrm{IC}_{50} 16.60 \mu \mathrm{M}\right)\end{array}$ & Jin et al. (2015) \\
\hline 15 & LPS-induced NO production in RAW264.7 cells $\left(\mathrm{IC}_{50} 5.94 \mu \mathrm{M}\right)$ & $\begin{array}{l}\text { Aminoguanidine } \\
\qquad\left(\mathrm{IC}_{50} 16.60 \mu \mathrm{M}\right)\end{array}$ & Jin et al. (2015) \\
\hline 18 & LPS-induced NO production in RAW264.7 cells $\left(\mathrm{IC}_{50} 16.66 \mu \mathrm{M}\right)$ & $\begin{array}{l}\text { Aminoguanidine } \\
\qquad\left(\mathrm{IC}_{50} 16.60 \mu \mathrm{M}\right)\end{array}$ & Jin et al. (2015) \\
\hline 23 & TNF- $\alpha$-stimulated A549 cells (IL-8 expression) & $\mathrm{NP}$ & $\begin{array}{l}\text { Ryu et al. } \\
\text { (2017) }\end{array}$ \\
\hline 26 & TNF- $\alpha$-stimulated A549 cells (IL- 8 expression) & NP & $\begin{array}{l}\text { Ryu et al. } \\
\text { (2017) }\end{array}$ \\
\hline 28 & LPS-induced NO production in RAW264.7 cells $\left(\mathrm{IC}_{50} 23.49 \mu \mathrm{M}\right)$ & $\begin{array}{l}\text { Aminoguanidine } \\
\qquad\left(\mathrm{IC}_{50} 16.60 \mu \mathrm{M}\right)\end{array}$ & Jin et al. (2015) \\
\hline 35 & TNF- $\alpha$-stimulated A549 cells (IL-8 expression) & NP & $\begin{array}{l}\text { Ryu et al. } \\
\quad(2017)\end{array}$ \\
\hline 38 & $\begin{array}{l}\text { LPS-induced THP-1 cells (TNF- } \alpha \text { expression; nuclear translocation of NF- } \kappa \text { B; } \\
\text { generation of ROS) }\end{array}$ & Prednisone & $\begin{array}{l}\text { Hanáková et al. } \\
\text { (2015) }\end{array}$ \\
\hline 52 & TNF- $\alpha$-stimulated A549 cells (IL- 8 expression) & NP & $\begin{array}{l}\text { Ryu et al. } \\
\quad(2017)\end{array}$ \\
\hline 53 & TNF- $\alpha$-stimulated A549 cells (IL-8 expression) & NP & $\begin{array}{l}\text { Ryu et al. } \\
\text { (2017) }\end{array}$ \\
\hline 54 & TNF- $\alpha$-stimulated A549 cells (IL- 8 expression) & NP & $\begin{array}{l}\text { Ryu et al. } \\
\quad(2017)\end{array}$ \\
\hline 56 & $\begin{array}{l}\text { LPS-induced THP-1 cells (TNF- } \alpha \text { expression; nuclear translocation of NF- } \kappa \mathrm{B} \text {; } \\
\text { generation of ROS) }\end{array}$ & Prednisone & $\begin{array}{l}\text { Hanáková et al. } \\
\text { (2015) }\end{array}$ \\
\hline
\end{tabular}

a Individual cell models, including RAW264.7 cells: murine macrophage cell line; J774.A1 cells: murine macrophage cell line; THP1 cells: monocytic leukaemia cell line; A549 cells: human alveolar basal epithelial adenocarcinoma cell line

b Individual enzymes and inflammatory cytokines, including COX-2: cyclooxygenase-2; MMP2: matrix metalloproteinase-2; ZFP36: zinc finger protein 36 ; NF- $\kappa$ B: nuclear factor $\kappa B$; ROS: reactive oxygen species; IL-8: interleukin 8 ; TNF- $\alpha$ : pro-inflammatory cytokine

${ }^{c}$ LPS: Lipopolysaccharide 
Table 5 Cytotoxic activity of different Paulownia C-geranylated flavonoids against human cancer cell lines in comparison to the standard cytotoxic agents

\begin{tabular}{|c|c|c|c|}
\hline Compounds & Cytotoxic effect ${ }^{\mathrm{a}}$ & Positive control & References \\
\hline \multirow[t]{2}{*}{1} & WB $344(\mathrm{NE})^{\mathrm{b}}$ & NP & Šmejkal et al. (2007) \\
\hline & THP-1 $\left(\mathrm{IC}_{50} 6.6 \mu \mathrm{M}\right)$ & NP & Hanáková et al. (2015) \\
\hline \multirow[t]{2}{*}{2} & WB $344\left(\mathrm{IC}_{50} 14.3 \mu \mathrm{M}\right)$ & NP & Šmejkal et al. (2007) \\
\hline & $\begin{array}{l}\text { MCF }\left(\mathrm{EC}_{50}<10 \mu \mathrm{M}\right) ; \mathrm{CEM}\left(\mathrm{EC}_{50} 3.2 \mu \mathrm{M}\right) \\
\text { RPMI826 }\left(\mathrm{EC}_{50}<10 \mu \mathrm{M}\right) ; \mathrm{U} 266\left(\mathrm{EC}_{50}\right. \\
2.4 \mu \mathrm{M}) ; \mathrm{HeLa}\left(\mathrm{EC}_{50}<10 \mu \mathrm{M}\right) ; \mathrm{BJ}\left(\mathrm{EC}_{50}\right. \\
5.9 \mu \mathrm{M}) ; \text { THP-1 }\left(\mathrm{EC}_{50}<10 \mu \mathrm{M}\right)\end{array}$ & $\begin{array}{l}\text { Olomoucine II, Diaziquone, } \\
\text { Oxaliplatin, Camptothecin } \\
\text { (Data in reference) }\end{array}$ & Šmejkal et al. (2010) \\
\hline 3 & $\begin{array}{l}\mathrm{MCF}\left(\mathrm{EC}_{50}<10 \mu \mathrm{M}\right) ; \mathrm{CEM}\left(\mathrm{EC}_{50}<10 \mu \mathrm{M}\right) ; \\
\text { RPMI8226 }\left(\mathrm{EC}_{50} 7.1 \mu \mathrm{M}\right) ; \mathrm{U} 266\left(\mathrm{EC}_{50}\right. \\
1.9 \mu \mathrm{M}) ; \mathrm{HeLa}\left(\mathrm{EC}_{50} 6.3 \mu \mathrm{M}\right) ; \mathrm{BJ}\left(\mathrm{EC}_{50}\right. \\
7.5 \mu \mathrm{M}) ; \text { THP-1 }\left(\mathrm{EC}_{50} 8.5 \mu \mathrm{M}\right)\end{array}$ & $\begin{array}{l}\text { Olomoucine II, Diaziquone, } \\
\text { Oxaliplatin, Camptothecin } \\
\text { (Data in reference) }\end{array}$ & Šmejkal et al. (2010) \\
\hline \multirow[t]{3}{*}{4} & WB $344\left(\mathrm{IC}_{50} 30.2 \mu \mathrm{M}\right)$ & $\mathrm{NP}$ & Šmejkal et al. (2007) \\
\hline & $\begin{array}{l}\text { MCF }\left(\mathrm{EC}_{50}<10 \mu \mathrm{M}\right) ; \mathrm{CEM}\left(\mathrm{EC}_{50}<10 \mu \mathrm{M}\right) \\
\text { RPMI8226 }\left(\mathrm{EC}_{50} 7.3 \mu \mathrm{M}\right) ; \mathrm{U} 266\left(\mathrm{EC}_{50}\right. \\
5.5 \mu \mathrm{M}) ; \mathrm{HeLa}\left(\mathrm{EC}_{50} 7.4 \mu \mathrm{M}\right) ; \mathrm{BJ}\left(\mathrm{EC}_{50}\right. \\
4.7 \mu \mathrm{M}) ; \text { THP-1 }\left(\mathrm{EC}_{50}<10 \mu \mathrm{M}\right)\end{array}$ & $\begin{array}{l}\text { Olomoucine II, Diaziquone, } \\
\text { Oxaliplatin, Camptothecin } \\
\text { (Data in reference) }\end{array}$ & Šmejkal et al. (2010) \\
\hline & THP-1 $\left(\mathrm{EC}_{50} 7.1 \mu \mathrm{M}\right)$ & NP & Hanáková et al. (2015) \\
\hline 6 & THP-1 $\left(\mathrm{IC}_{50} 7.9 \mu \mathrm{M}\right)$ & $\mathrm{NP}$ & Hanáková et al. (2015) \\
\hline 8 & THP-1 ( $\left.\mathrm{IC}_{50} 6.4 \mu \mathrm{M}\right)$ & NP & Hanáková et al. (2015) \\
\hline 10 & THP-1 $\left(\mathrm{IC}_{50} 8.0 \mu \mathrm{M}\right)$ & NP & Hanáková et al. (2015) \\
\hline 15 & THP-1 $\left(\mathrm{IC}_{50} 7.2 \mu \mathrm{M}\right)$ & $\mathrm{NP}$ & Hanáková et al. (2015) \\
\hline 19 & $\mathrm{~A} 549\left(\mathrm{IC}_{50} 8.9 \mu \mathrm{M}\right)$ & Oridonin $\left(\mathrm{IC}_{50} 18.7 \mu \mathrm{M}\right)$ & Gao et al. (2015) \\
\hline 27 & $\mathrm{~A} 549\left(\mathrm{IC}_{50} 23.7 \mu \mathrm{M}\right)$ & Oridonin $\left(\mathrm{IC}_{50} 18.7 \mu \mathrm{M}\right)$ & Gao et al. (2015) \\
\hline 39 & THP-1 ( $\left.\mathrm{IC}_{50} 6.5 \mu \mathrm{M}\right)$ & $\mathrm{NP}$ & Hanáková et al. (2015) \\
\hline 40 & THP-1 $\left(\mathrm{IC}_{50}>5 \mu \mathrm{M}\right)$ & NP & Kollár et al. (2011) \\
\hline 56 & THP-1 ( $\left.\mathrm{IC}_{50} 6.7 \mu \mathrm{M}\right)$ & NP & Hanáková et al. (2015) \\
\hline 60 & $\mathrm{~A} 549\left(\mathrm{IC}_{50} 22.1 \mu \mathrm{M}\right)$ & Oridonin $\left(\mathrm{IC}_{50} 18.7 \mu \mathrm{M}\right)$ & Gao et al. (2015) \\
\hline 64 & THP-1 ( $\left.\mathrm{IC}_{50} 6.7 \mu \mathrm{M}\right)$ & NP & Hanáková et al. (2015) \\
\hline
\end{tabular}

a Individual cell models, including WB 344: epithelioid cell line; A549: lung cancer cell line; MCF-7: breast carcinoma cell line; CEM: T-lymphoblastic leukaemia cell line; RPMI 8226 and U266: multiple myeloma cell line; HeLa: cervical cancer cell line; THP1: monocytic leukemia cell line; BJ: fibroblast cell line

b NE: The $\mathrm{IC}_{50}$ could not be estimated because the cytotoxicity was less than $50 \%$ of that of the control

The cholinestrase inhibitory effects of nine Paulownia C-geranylated flavonoids (1, 2, 4, 7, 9, 11, 13, 15 and 16) were estimated and all of them apart from 11 exhibited cholinesterase inhibition with $\mathrm{IC}_{50}$ values ranging from 7.2 to $316 \mu \mathrm{M}$ for human acetylcholinesterase (hAChE) and 1.4-80.0 $\mu \mathrm{M}$ for butyrylcholinestrase (BChE). Diplacone (2) was the most effective inhibitor ( $\mathrm{IC}_{50} 7.2$ and $1.4 \mu \mathrm{M}$ ), compared with $\mathrm{IC}_{50} 0.15$ and $3.7 \mu \mathrm{M}$ for the positive control, eserine (Cho et al. 2012).

Twelve Paulownia $C$-geranylated flavanones $(\mathbf{1}, \mathbf{2}$, $4,7, \mathbf{1 1}, \mathbf{1 3}, \mathbf{1 5}, \mathbf{4 7 - 5 1}$ ) were selected to examine their inhibition of severe acute respiratory syndrome-CoV papain-like protease (SARS-CoV PLpro) and all were active in a dose-dependent manner with $\mathrm{IC}_{50}$ values ranging between 5.0 and $14.4 \mu \mathrm{M}$. Those $C$-geranylated flavanones with a 3,4-dihydro- $2 \mathrm{H}$-pyran moiety (47-51) showed better inhibition than their parent compounds, and $\mathbf{4 8}$ was considered as a reversible inhibitor with a $\mathrm{y}$ axis intercept of 0 and $\mathrm{IC}_{50}$ of $6.1 \mu \mathrm{M}$ (Cho et al. 2013).

Moreover, the antiparasitic activities of some Paulownia $C$-geranylated flavonoids were further evaluated by molecular docking energy analysis to 
Table 6 Enzymatic inhibitory effects of different Paulownia $C$-geranylated flavonoids in comparison to the positive controls

\begin{tabular}{|c|c|c|c|}
\hline Compounds & Enzymatic inhibitory effects ${ }^{\mathrm{a}}\left(\mathrm{IC}_{50}, \mu \mathrm{M}\right)$ & Positive control & References \\
\hline \multirow[t]{3}{*}{1} & COX-1 (3.7); COX-2 (6.0) & Ibuprofen $\left(\mathrm{IC}_{50} 6.3,4.2 \mu \mathrm{M}\right)$ & Hanáková et al. (2017) \\
\hline & SARS-CoV PLpro (14.4) & NP & Cho et al. (2013) \\
\hline & PTP1B (1.9); $\alpha$-Glucosidase (30.7) & $\mathrm{NaVO}_{4}\left(\mathrm{IC}_{50} 32.6 \mu \mathrm{M}\right) ;$ Voglibose $\left(\mathrm{IC}_{50} 24.5 \mu \mathrm{M}\right)$ & Song et al. (2017) \\
\hline \multirow[t]{3}{*}{2} & COX-1 (1.8), COX-2 (4.2); 5-LOX (0.05) & Ibuprofen $(6.3,4.2)$; Zileuton $(0.35)$ & Hanáková et al. (2017) \\
\hline & $\operatorname{AChE~(7.2),~BChE~(1.4)~}$ & Eserine $(0.15,3.7)$ & Cho et al. (2012) \\
\hline & SARS-CoV PLpro (10.4) & $\mathrm{NP}$ & Cho et al. (2013) \\
\hline \multirow[t]{3}{*}{4} & HNE (7.8) & Luteolin (12.7) & Ryu et al. (2017) \\
\hline & SARS-CoV PLpro (13.2) & NP & Cho et al. (2013) \\
\hline & PTP1B (3.9); $\alpha$-Glucosidase (18.4) & $\mathrm{NaVO}_{4}$ (32.6); Voglibose (24.5) & Song et al. (2017) \\
\hline 5 & $\begin{array}{l}\text { COX-1 (3.3), COX-2 (10.6); 5-LOX } \\
\quad(0.06)\end{array}$ & Ibuprofen $(6.3,4.2)$; Zileuton $(0.35)$ & Hanáková et al. (2017) \\
\hline 6 & $5-\operatorname{LOX}(0.38)$ & Zileuton (0.35) & Hanáková et al. (2017) \\
\hline \multirow[t]{2}{*}{7} & SARS-CoV PLpro (12.7) & NP & Cho et al. (2013) \\
\hline & PTP1B (7.8); $\alpha$-Glucosidase (19.6) & $\mathrm{NaVO}_{4}$ (32.6); Voglibose (24.5) & Song et al. (2017) \\
\hline \multirow[t]{2}{*}{8} & COX-1 (4.2), COX-2 (6.4) & Ibuprofen $(6.3,4.2)$ & Hanáková et al. (2017) \\
\hline & PTP1B (3.8); $\alpha$-Glucosidase (78.9) & $\mathrm{NaVO}_{4}$ (32.6); Voglibose (24.5) & Song et al. (2017) \\
\hline \multirow[t]{3}{*}{9} & HNE (3.3) & Luteolin (12.7) & Ryu et al. (2017) \\
\hline & AChE (22.9), BChE (6.4) & Eserine $(0.15,3.7)$ & Cho et al. (2012) \\
\hline & PTP1B (5.9); $\alpha$-Glucosidase (6.5) & $\mathrm{NaVO}_{4}$ (32.6); Voglibose (24.5) & Song et al. (2017) \\
\hline 11 & SARS-CoV PLpro (13.9) & $\mathrm{NP}$ & Cho et al. (2013) \\
\hline \multirow[t]{3}{*}{13} & AChE (31.9), BChE (12.7) & Eserine $(0.15,3.7)$ & Cho et al. (2012) \\
\hline & SARS-CoV PLpro (9.2) & NP & Cho et al. (2013) \\
\hline & PTP1B (8.2); $\alpha$-Glucosidase (25.8) & $\mathrm{NaVO}_{4}$ (32.6); Voglibose (24.5) & Song et al. (2017) \\
\hline \multirow[t]{3}{*}{15} & AChE (48.5), BChE (11.2) & Eserine $(0.15,3.7)$ & Cho et al. (2012) \\
\hline & SARS-CoV PLpro (9.2) & $\mathrm{NP}$ & Cho et al. (2013) \\
\hline & PTP1B (4.9); $\alpha$-Glucosidase (17.8) & $\mathrm{NaVO}_{4}$ (32.6); Voglibose (24.5) & Song et al. (2017) \\
\hline \multirow[t]{2}{*}{16} & AChE (15.6), BChE (3.8) & Eserine $(0.15,3.7)$ & Cho et al. (2012) \\
\hline & PTP1B (6.6); $\alpha$-Glucosidase (2.2) & $\mathrm{NaVO}_{4}$ (32.6); Voglibose (24.5) & Song et al. (2017) \\
\hline 21 & HNE (6.7) & Luteolin (12.7) & Ryu et al. (2017) \\
\hline 23 & HNE (6.3) & Luteolin (12.7) & Ryu et al. (2017) \\
\hline 25 & HNE (2.4) & Luteolin (12.7) & Ryu et al. (2017) \\
\hline 30 & HNE (15.4) & Luteolin (12.7) & Ryu et al. (2017) \\
\hline 35 & HNE (13.6) & Luteolin (12.7) & Ryu et al. (2017) \\
\hline 47 & $\mathrm{HNE}(8.4)$ & Luteolin (12.7) & Ryu et al. (2017) \\
\hline 48 & SARS-CoV PLpro (6.2) & NP & Cho et al. (2013) \\
\hline 49 & SARS-CoV PLpro (6.1) & $\mathrm{NP}$ & Cho et al. (2013) \\
\hline 50 & SARS-CoV PLpro (11.6) & NP & Cho et al. (2013) \\
\hline 51 & SARS-CoV PLpro (5.0) & NP & Cho et al. (2013) \\
\hline 57 & $5-\operatorname{LOX}(0.35)$ & Zileuton (0.35) & Hanáková et al. (2017) \\
\hline 60 & 5-LOX (0.37) & Zileuton (0.35) & Hanáková et al. (2017) \\
\hline
\end{tabular}

${ }^{a}$ Individual enzyme models, including COX-1 and COX-2: cyclooxygenase-1 and 2; 5-LOX: 5-lipoxygenase; SARS-CoV PLpro: severe acute respiratory syndrome-CoV papain-like protease; PTP1B: protein tyrosine phosphatase 1B; hAChE: human acetylcholinestrase; BChE: butyrylcholinestrase; HNE: human neutrophil elastase 
identify potential protein targets of Leishmania enzymes. Docking energies (value of Edock) for diplacone (2) to L. major $N$-myristoyltransferase ($135.7 \mathrm{~kJ} / \mathrm{mol}$ ); 3'-O-methyl diplacone (4) to $L$. pteridine reductase $1(-142.2 \mathrm{~kJ} / \mathrm{mol})$, to $L$. glycerol-3-phosphate dehydrogenase $(-148.3 \mathrm{~kJ} / \mathrm{mol})$ and to $L$. cyclophilin $(-126.5 \mathrm{~kJ} / \mathrm{mol}) ; 4^{\prime}-O$-methyldiplacone (7) to $L$. glycerol-3-phosphate dehydrogenase ($143.1 \mathrm{~kJ} / \mathrm{mol}$ ); and $3^{\prime}-O$-methyldiplacol (15) to $L$. methionyl-tRNA synthetase $(-145.9 \mathrm{~kJ} / \mathrm{mol})$, have demonstrated their antiparasitic activities against both L. donovani and T. b. brucei (Ogungbe et al. 2014).

In addition, the inhibition of cyclooxygenases (COX-1 and COX-2) and 5-lipoxygenase (5-LOX) for nine Paulownia $C$-geranylated flavonoids was performed to test their anti-inflammatory effects. Compounds 1, 2, 5, 8 and 59 showed significant effects with $\mathrm{IC}_{50}$ values ranging from 1.8 to $26.3 \mu \mathrm{M}$ for COX-1 and from 4.2 to $10.6 \mu \mathrm{M}$ for COX-2, which were better than the reference inhibitor ibuprofen ( $\mathrm{IC}_{50} 6.3$ and $4.2 \mu \mathrm{M}$ ). Diplacone (2) was the best inhibitor $\left(\mathrm{IC}_{50} 1.8\right.$ and $\left.4.2 \mu \mathrm{M}\right)$ but with no selectivity for COX-1 and COX-2 (the COX-1/COX2 selectivity ratio was 0.43 ). Although 59 showed weaker inhibition ( $\mathrm{IC}_{50} 26.3$ and $\left.9.5 \mu \mathrm{M}\right)$, it possessed greater selectivity against COX-2 (COX$1 / \mathrm{COX}-2$ selectivity ratio was 2.8 ). Meanwhile, compounds 2, 5, 6, 57, and 60 were selected for determination of their effect on 5-LOX. Compound $2\left(\mathrm{IC}_{50} 0.05 \mu \mathrm{M}\right)$ and $5\left(\mathrm{IC}_{50} 0.06 \mu \mathrm{M}\right)$ showed activities almost 10 times greater than that of zileuton $\left(\mathrm{IC}_{50} 0.35 \mu \mathrm{M}\right)$ as the positive control (Hanáková et al. 2017). Furthermore, compounds 4, 9, 21, 23, 25 and 47 were found to greatly inhibit human neutrophil elastase (HNE) activity with $\mathrm{IC}_{50}$ values ranging from 2.4 to $8.4 \mu \mathrm{M}$ and compound 9 was selected as the best potential inhibitor by kinetic enzymatic assays with the HNE substrate MeOSucAAPV-pNA (Ryu et al. 2017).

The methanol extract of $P$. tomentosa fruits was characterized as a source of PTP1B and $\alpha$-glucosidase inhibitors and eight isolated $C$-geranylated flavonoids $(1,4,6,7,9,13,15$ and 16) displayed dual inhibition of PTP1B and $\alpha$-glucosidase. The inhibitory potency of these compounds varied accordingly, but most of the compounds were more effective against PTP1B than $\alpha$-glucosidase. Mimulone (1) was the most effective against PTP1B with
$\mathrm{IC}_{50} 1.9 \mu \mathrm{M}\left(\mathrm{NaVO}_{4}\right.$ as the positive control, $\mathrm{IC}_{50}$ $32.6 \mu \mathrm{M}$ ), whereas 6-geranyl-3,3',5,5',7-pentahydroxy-4'-methoxyflavane (16) displayed potent inhibition against an $\alpha$-glucosidase with $\mathrm{IC}_{50}$ $2.2 \mu \mathrm{M}$ compared with $\mathrm{IC}_{50} 24.5 \mu \mathrm{M}$ for the reference inhibitor, Voglibose (Song et al. 2017).

Neuroprotective effects

The neuroprotective effects of mimulone (1) and diplacone (2) against glutamate-induced neurotoxicity were studied in primary cultured rat cortical cells. It was found that only diplacone (2) weakly attenuated glutamate-induced toxicity at $10 \mu \mathrm{M}$ (Kim et al. 2010).

\section{Structure-activity relationship (SAR) of Paulownia C-geranylated flavonoids}

Possible SARs involved in the radical scavenging of flavonoids have been discussed carefully (Cao et al. 1997; Dugas et al. 2000; Sekher et al. 2001; Heim et al. 2002; Zheng et al. 2019), however, it could be considered that the substitution of a geranyl group or its oxidized congeners did not significantly alter the radical scavenging activity of Paulownia $C$-geranylated flavonoids (Smejkal et al. 2007; Asai et al. 2008; Wang et al. 2017). Regarding cellular biological activities (anti-inflammatory and cytotoxic effects), antibacterial and antiparasitic activities, and enzymatic inhibitions, an ortho-dihydroxy arrangement at $\mathrm{C}-3^{\prime}, 4^{\prime}$ of the B-ring was considered an essential group but also enhanced the cytotoxic risk. Hydroxylation at C-3 in ring $\mathrm{C}$ and 4'-methoxy substitution of ring B might cause a loss of bioactive potency (Šmejkal et al. 2008a; Wang et al. 2019). In addition, the unmodified geranyl group at the C-6 position seemed to be crucial for these various biological effects in vitro and in vivo (Vochyánová et al. 2015) of $C$-geranylated flavonoids, however, hydroxylation on the distal end of the geranyl substituent decreased the biological activities revealed by different research (Alcaráz et al. 2000; Šmejkal et al. 2010; Hanáková et al. 2017; Ryu et al. 2017). The presence of a $\beta$-carbon (proximal) $\mathrm{OH}$ group on the geranyl chain did not affect the cytotoxicity (Hanáková et al. 2015). In addition, the 3,4-dihydro-2H-pyran moiety in some Paulownia 
C-geranylated flavonoids seemed to be positive for their SARS-CoV PLpro enzyme inhibition (Cho et al. 2013).

\section{Research and application prospects of Paulownia $C$-geranylated flavonoids}

Phytochemical research demonstrated that Paulownia species are natural resources of $C$-geranylated flavonoids with varied geranyl substituents. There might be another $C$-geranylated flavonoid constituent with more attractive geranyl variations, such as the cyclic monoterpene side-chain in paucatalinone $\mathrm{F}$ (67, Wang et al. 2019), metabolized in Paulownia species. Theirfore, it is worthwhile to carry out further investigations on Paulownia species for more novel $C$-geranylated flavonoids on the phytochemical view.

Moreover, it was reported that the $C$-isoprenoid chain could increase the affinity of flavonoids towards P-glycoprotein (P-gp) located at the cell membrane because of the increased hydrophobicity (Barron et al. 2002). Some Paulownia C-geranylated flavonoids were supposed to have a greater ability to penetrate the membranes of cells than a lipophilic substituent (Tsuchiya and Iinuma 2000; Šmejkal 2014). We considered the possibility that different modifications of geranyls in $C$-geranylated flavonoids could influence their transmembrane absorption or intracellular distribution. Unfortunately, almost none of the Paulownia $C$-geranylated flavonoids had been assayed to evaluate these correlative physiological effects. The role of diverse geranyl variations in Paulownia $C$-geranylated flavonoids in these physiological effects should be worth evaluating in the further studies.

Among the Paulownia $C$-geranylated flavonoids, diplacone (2), first isolated from Diplacus aurantiacus (Lincoln 1980), was a main component with a high content in the flower and fruit of $P$. tomentosa (Jiang et al. 2004; Chen et al. 2009; Holubová and Šmejkal 2011). Recently, a series of biological activities such as those mentioned above were evaluated for this compound. The results indicated that it possessed excellent antioxidant and antiinflammatory effects, and it was considered as a potential antioxidant therapy agent for the treatment of inflammatory bowel disease (Moura et al. 2015) and as a potential novel 5-lipoxygenase inhibitor for the treatment of asthma (Bruno et al. 2018). At present, diplacone and its isomers have been protected by patent application as a pharmaceutical composition for treating an ocular disease (Liao 2016). Diplacone may be used as a lead compound for new drug design.

\section{Conclusions}

The article presents an overview of $C$-geranylated flavonoids from Paulownia species focusing on their structural variety, key spectroscopic characteristics, biological activity with structure-activity relationships and application prospects. To date, 76 naturally occurring Paulownia $C$-geranylated flavonoids have been reported in the phytochemical literature. Among them, $C$-geranylflavanones were predominant constituents with a single geranyl substituent mainly attached to the C-6 position of the flavonoid skeleton. Furthermore, the geranyl substituent could suffer different modifications, such as oxidation (hydroxylation and carbonylation), dehydration, cyclization, or special reduction, to result in the structural variety and novelty of Paulownia $C$-geranylflavonoids. As a natural resource, fruits of $P$. tomentosa were explored more abundantly and $P$. catalpifolia gave rise to some unusual C-geranylflavonoids. Unfortunately, other Paulownia species were with poor attention by researchers and need further phytochemical and pharmacological investigations.

Meanwhile, Paulownia C-geranylflavonoids displayed a wide spectrum of biological activities associated with their structural varieties and their antioxidant and anti-inflammatory activities were the focus of pharmacological research. Given the traditional medicinal use of Paulownia plants, it is worthwhile to evaluate some Paulownia $C$-geranylflavonoids on some airway inflammation diseases, such as chronic bronchitis and asthma.

In brief, structural variations in Paulownia $C$ geranylflavonoids with excellent biological activities suggested this kind of constituent might be worthy of further study and may be valuable for the development of new drug candidates.

Acknowledgements This work was financially supported by the Innovation Project of Shandong Academy of Medical 
Sciences (2019), the Science Development Project of Shandong Academy of Medical Science (2015-08) and the Natural Science Open Foundation of State Key Laboratory of Bioactive Substances and Function of Natural Medicines (GTZK201503).

\section{References}

Alcaráz LE, Blanco SE, Puig ON et al (2000) Antibacterial activity of flavonoids against methicillin-resistant Staphylococcus aureus strains. J Theor Biol 205:231-240

Andersen OM, Markham KR (2006) Flavonoids: chemistry, biochemistry, applications. CRC Press, Boca Raton

Asai T, Hara N, Kobayashi S et al (2008) Geranylated flavanones from the secretion on the surface of the immature fruits of Paulownia tomentosa. Phytochemistry 69:12341241

Barron D, Ibrahim RK (1996) Isoprenylated flavonoids-a survey. Phytochemistry 43:921-982

Barron D, Pietro AD, Dumontet C et al (2002) Isoprenoid flavonoids are new leads in the modulation of chemoresistance. Phytochem Rev 1:325-332

Bergmann BA (1998) Propagation method influences first year field survival and growth of Paulownia. New Forest 16:251-264

Bruno F, Spaziano G, Liparulo A et al (2018) Recent advances in the search for novel 5-lipoxygenase inhibitors for the treatment of asthma. Eur J Med Chem 153:65-72

Cao G, Sofic E, Prior RL (1997) Antioxidant and prooxidant behavior of flavonoids: structure-activity relationships. Free Radical Biol Med 22:749-760

Chen J, Liu Y, Shi YP (2009) Determination of flavonoids in the flowers of Paulownia tomentosa by high-performance liquid chromatography. J Anal Chem 64:282-288

Chen R, Gao BQ, Liu X et al (2017a) Molecular insights into the enzyme promiscuity of an aromatic prenyltransferase. Nat Chem Biol 13:226-234

Chen HN, Tang WZ, Yan ZH et al (2017b) Protective effect of diplacone on vascular endothelial injury induced by homocysteine. Chin J Arterioscler 25:666-670

Chinese Flora Editorial Committee (1998) Flora of China, Paulownia Siebold \& Zuccarini. Chinese Academy of Sciences, Beijing, http://foc.iplant.cn/search.aspx?k= Paulownia\&id=1

Cho JK, Ryu YB, Curtis-Long MJ et al (2012) Cholinestrase inhibitory effects of geranylated flavonoids from Paulownia tomentosa fruits. Bioorgan Med Chem 20:2595-2602

Cho JK, Curtis-Long MJ, Lee KH et al (2013) Geranylated flavonoids displaying SARS-CoV papain-like protease inhibition from the fruits of Paulownia tomentosa. Bioorg Med Chem 21:3051-3057

Duan WD, Zhang J, Xie G et al (2007) Chemical constituents from the flower of Paulownia fortune (Seem) Hemsl. J Chin Med Mater 30:168-170

Dugas AJ, Castaneda-Acosta J, Bonin GC et al (2000) Evaluation of the total peroxyl radical scavenging capacity of flavonoids: structure-activity relationships. J Nat Prod 63:327-331
Erbar C, Gülden C (2011) Ontogeny of the flowers in Paulownia tomentosa-a contribution to the recognition of the resurrected monogeneric family Paulowniaceae. Flora 206:205-218

Gang DR, Beuerle T, Ullmann P et al (2002) Differential production of meta hydroxylated phenylpropanoids in sweet basil peltate glandular trichomes and leaves is controlled by the activities of specific acyltransferases and hydroxylases. Plant Physiol 130:1536-1544

Gao TY, Jin X, Tang WZ et al (2015) New geranylated flavanones from the fruits of Paulownia catalpifolia Gong Tong with their anti-proliferative activity on lung cancer cells A549. Bioorgan Med Chem Lett 25:3686-3689

Hanáková Z, Hošek J, Babula P et al (2015) C-Geranylated flavanones from Paulownia tomentosa fruits as potential anti-inflammatory compounds acting via inhibition of TNF- $\alpha$ production. J Nat Prod 78:850-863

Hanáková Z, Hošek J, Kutil Z et al (2017) Anti-inflammatory activity of natural geranylated flavonoids: cyclooxygenase and lipoxygenase inhibitory properties and proteomic analysis. J Nat Prod 80:999-1006

He T, Vaidya BN, Perry ZD et al (2016) Paulownia as a medicinal tree: traditional uses and current advances. Eur J Med Plants 14:1-15

Heim KE, Tagliaferro AR, Bobilya DJ (2002) Flavonoid antioxidants: chemistry, metabolism and structure activity relationships. J Nutr Biochem 13:572-584

Holubová P, Smejkal K (2011) Changes in the level of bioactive compounds in Paulownia tomentosa fruits. J Liq Chromatogr Relat Technol 34:276-288

Hošek J, Závalová V, Šmejkal K et al (2010) Effect of diplacone on LPS-induced inflammatory gene expression in macrophages. Folia Biol (Praha) 56:124-130

Hošek J, Toniolo A, Neuwirth O et al (2013) Prenylated and geranylated flavonoids increase production of reactive oxygen species in mouse macrophages but inhibit the inflammatory response. J Nat Prod 76:1586-1591

Hsu CL, Shyu MH, Lin JA et al (2011) Cytotoxic effects of geranyl flavonoid derivatives from the fruit of Artocarpus communisin SK-Hep-1 human hepatocellular carcinoma cells. Food Chem 127:127-134

Huang YL, Yeh PY, Shen CC et al (2003) Antioxidant flavonoids from the rhizomes of Helminthostachys zeylanica. Phytochemistry 64:1277-1283

Huang YC, Hwang TL, Yang YL et al (2010) Acetogenin and prenylated flavonoids from Helminthostachys zeylanica with inhibitory activity on superoxide generation and elastase release by neutrophils. Planta Med 76:447-453

Jiang TF, Du X, Shi YP (2004) Determination of flavonoids from Paulownia tomentosa (Thunb) Steud. by micellar electrokinetic capillary electrophoresis. Chromatographia 59:255-258

Jin Q, Lee C, Lee JW et al (2015) Geranylated flavanones from Paulownia coreana and their inhibitory effects on nitric oxide production. Chem Pharm Bull 63:384-387

Kim SK, Cho SB, Moon HI (2010) Neuroprotective effects of a sesquiterpene lactone and flavanones from Paulownia tomentosa Steud. against glutamate-induced neurotoxicity in primary cultured rat cortical cells. Phytother Res 24:1898-1900 
Kobayashi S, Asai T, Fujimoto Y et al (2008) Anti-herbivore structures of Paulownia tomentosa: morphology, distribution, chemical constituents and changes during shoot and leaf development. Ann Bot 101:1035-1047

Kollár P, Bárta T, Závalová V et al (2011) Geranylated flavanone tomentodiplacone $\mathrm{B}$ inhibits proliferation of human monocytic leukaemia (THP-1) cells. Brit J Pharmacol 162:1534-1541

Kuzuyama T, Noel JP, Richard SB (2005) Structural basis for the promiscuous biosynthetic prenylation of aromatic natural products. Nature 435:983-987

Li XQ, Wu JL, Cao FH et al (2008) Chemical constituents from leaves of Paulownia fortunei. J Chin Med Mater $31: 850-852$

Liao CC (2016) Compounds for treating ocular diseases. US Patent 9,370,503 B1, 21 Jun 2016

Lin JA, Wu CH, Fang SC et al (2012) Combining the observation of cell morphology with the evaluation of key inflammatory mediators to assess the anti-inflammatory effects of geranyl flavonoid derivatives in bread fruit. Food Chem 132:2118-2125

Lincoln DE (1980) Leaf resin flavonoids of Diplacus aurantiacus. Biochem Syst Ecol 8:397-400

Mabry TJ, Markham KR, Thomas MB (1969) The systematic identification of flavonoids. Springer, New York

Moon HI, Jeong MH, Jo WS (2014) Protective activity of $C$ geranylflavonoid analogs from Paulownia tomentosa against DNA damage in $137 \mathrm{Cs}$ irradiated AHH-1 cells. Nat Prod Commun 9:1295-1298

Moura FA, de Andrade KQ, dos Santos JCF et al (2015) Antioxidant therapy for treatment of inflammatory bowel disease: does it work. Redox Bio 6:617-639

Navrátilová A, Schneiderová K, Veselá D et al (2013) Minor $C$-geranylated flavanones from Paulownia tomentosa fruits with MRSA antibacterial activity. Phytochemistry 89:104-113

Navrátilová A, Nešuta O, Vančatová I et al (2016) C-Geranylated flavonoids from Paulownia tomentosa fruits with antimicrobial potential and synergistic activity with antibiotics. Pharm Biol 54:1398-1407

Ogungbe IV, Erwin WR, Setzer WN (2014) Antileishmanial phytochemical phenolics: molecular docking to potential protein targets. J Mol Graph Model 48:105-117

Ríos JL, Recio MC (2005) Medicinal plants and antimicrobial activity. J Ethnopharmacol 100:80-84

Ryu HW, Park YJ, Lee SU et al (2017) Potential anti-inflammatory effects of the fruits of Paulownia tomentosa. J Nat Prod 80:2659-2665

Salem MM, Capers J, Rito S et al (2011) Antiparasitic activity of C-geranyl flavonoids from Mimulus bigelovii. Phytother Res 25:1246-1249

Schneiderová K, Šmejkal K (2014) Phytochemical profile of Paulownia tomentosa (Thunb). Phytochem Rev, Steud. https://doi.org/10.1007/s11101-014-9376-y

Schneiderová K, Ślapetová T, Hrabal R et al (2013) Tomentomimulol and mimulone B: two new C-geranylated flavonoids from Paulownia tomentosa fruits. Nat Prod Res 27:613-618
Sekher PA, Chan TS, O'Brien PJ et al (2001) Flavonoid B-ring chemistry and antioxidant activity: fast reaction kinetics. Biochem Biophys Res Commun 282:1161-1168

Slade D, Ferreira D, Marais JPJ (2005) Circular dichroism, a powerful tool for the assessment of absolute configuration of flavonoids. Phytochemistry 66:2177-2215

Šmejkal K (2014) Cytotoxic potential of $C$-prenylated flavonoids. Phytochem Rev 13:245-275

Smejkal K, Holubova P, Zima A et al (2007) Antiradical activity of Paulownia tomentosa (Scrophulariaceae) extracts. Molecules 12:1210-1219

Šmejkal K, Grycová L, Marek R et al (2007) C-Geranyl Compounds from Paulownia tomentosa fruits. J Nat Prod 70:1244-1248

Šmejkal K, Chudík S, Klouček P et al (2008a) Antibacterial $C$ geranylflavonoids from Paulownia tomentosa fruits. J Nat Prod 71:706-709

Šmejkal K, Babula P, Šlapetová T et al (2008b) Cytotoxic activity of $C$-geranyl compounds from Paulownia tomentosa fruits. Planta Med 74:1488-1491

Šmejkal K, Svačinová J, Šlapetová T et al (2010) Cytotoxic activities of several geranyl-substituted flavanones. J Nat Prod 73:568-572

Song YH, Uddin Z, Jin YM et al (2017) Inhibition of protein tyrosine phosphatase (PTP1B) and $\alpha$-glucosidase by geranylated flavonoids from Paulownia tomentosa. J Enzyme Inhib Med Chem 32:1195-1202

Tang WZ, Wang YA, Gao TY et al (2017) Identification of $C$ geranylated flavonoids from Paulownia catalpifolia Gong Tong fruits by HPLC-DAD-ESI-MS/MS and their antiaging effects on $2 \mathrm{BS}$ cells induced by $\mathrm{H}_{2} \mathrm{O}_{2}$. Chin $\mathrm{J}$ Nat Med 15:384-391

Tsuchiya H, Iinuma M (2000) Reduction of membrane fluidity by antibacterial sophoraflavanone $\mathrm{G}$ isolated from Sophora exigua. Phytomedicine 7:161-165

Vochyánová Z, Bartošová L, Bujdáková V et al (2015) Diplacone and mimulone ameliorate dextran sulfate sodiuminduced colitis in rats. Fitoterapia 101:201-207

Wang YA, Xue J, Jia XH et al (2017) Chemical constituents from the fruit peel of Paulownia catalpifolia. J Chin Med Mater 40:1591-1595

Wang YA, Guo X, Jia XH et al (2019) Undescribed $C$-geranylflavonoids isolated from the fruit peel of Paulownia catalpifolia T. Gong ex D.Y. Hong with their protection on human umbilical vein endothelial cells injury induced by hydrogen peroxide. Phytochemistry 158:126-134

Xing YL, Bi LW, Zhao ZD et al (2013) Research progress in plant resources and chemistry of Paulownia. Chem Ind For Prod 33:135-140

Yazaki K, Sasaki K, Tsurumaru Y (2009) Prenylation of aromatic compounds, a key diversification of plant secondary metabolites. Phytochemistry 70:1739-1745

Zhang PF, Li C (2008) Flavones from flowers of Paulownia fortune. China J Chin Mater Med 33:2629-2632

Zheng YZ, Deng G, Chen DF et al (2019) The influence of $\mathrm{C}_{2}=\mathrm{C}_{3}$ double bond on the antiradical activity of flavonoid: different mechanisms analysis. Phytochemistry 157:1-7 
Zhu ZH, Chao CJ, Lu XY (1986) Paulownia in China: cultivation and utilization. Asian Network for Biological Science and International Development Research Centre, Chinese Academy of Forestry, Beijing

Zima A, Hošek J, Treml J et al (2010) Antiradical and cytoprotective activities of several $C$-geranyl-substituted flavanones from Paulownia tomentosa fruit. Molecules 15:6035-6049

Publisher's Note Springer Nature remains neutral with regard to jurisdictional claims in published maps and institutional affiliations. 